\title{
ARCHIMEDEAN COHOMOLOGY REVISITED
}

\author{
CATERINA CONSANI AND MATILDE MARCOLLI $^{\dagger}$
}

\section{INTRODUCTION}

C. Deninger produced a unified description of the local factors at arithmetic infinity and at the finite places where the local Frobenius acts semi-simply, in the form of a Ray-Singer determinant of a "logarithm of Frobenius" $\Phi$ on an infinite dimensional vector space (the archimedean cohomology $H_{a r}(X)$ at the archimedean places, $c f$. [11]). The first author gave a cohomological interpretation of the space $H_{a r}(X)$, in terms of a double complex $K^{\prime, \cdot}$ of real differential forms on a smooth projective algebraic variety $X$ (over $\mathbb{C}$ or $\mathbb{R}$ ), with Tate-twists and suitable cutoffs, together with an endomorphism $N$, which represents a "logarithm of the local monodromy at arithmetic infinity". Moreover, in this theory the cohomology of the complex Cone $(N)^{\cdot}$ computes real Deligne cohomology of $X(c f .9$ ). The construction of [9] is motivated by a dictionary of analogies between the geometry of the tubular neighborhoods of the "fibers at arithmetic infinity" of an arithmetic variety $X$ and the geometric theory of the limiting mixed Hodge structure of a degeneration over a disk. Thus, the formulation and notation used in 9 for the double complex and archimedean cohomology mimics the definition, in the geometric case, of a resolution of the complex of nearby cycles and its cohomology (cf. [28]).

In Section 2 and 3 we give an equivalent description of Consani's double complex, which allows us to investigate further the structure induced on the complex and the archimedean cohomology by the operators $N, \Phi$, and the Lefschetz operator $\mathbb{L}$. In Section 4 we illustrate the analogies between the complex and archimedean cohomology and a resolution of the complex of nearby cycles in the classical geometry of an analytic degeneration with normal crossings over a disk. In Section 5 we show that, using the Connes-Kreimer formalism of renormalization, we can identify the endomorphism $N$ with the residue of a Fuchsian connection, in analogy to the log of the monodromy in the geometric case. In Section 6 we recall Deninger's approach to the archimedean cohomology through an interpretation as global sections of a real analytic Rees sheaf over $\mathbb{R}$. In Section 7 we show how the action of the endomorphisms $N$ and $\mathbb{L}$ and the Frobenius operator $\Phi$ define a noncommutative manifold (a spectral triple in the sense of Connes), where the algebra is related to the $\mathrm{SL}(2, \mathbb{R})$ representation associated to the Lefschetz $\mathbb{L}$, the Hilbert space is obtained by considering Kernel and Cokernel of powers of $N$, and the $\log$ of Frobenius $\Phi$ gives the Dirac operator. The archimedean part of the Hasse-Weil L-function is obtained from a zeta function of the spectral triple. In Section 8 we outline some formal analogies between the complex and cohomology at arithmetic infinity and the equivariant Floer cohomology of loop spaces considered in Givental's homological geometry of mirror symmetry.

\section{Cohomology at ARIthmetic infinity}

Let $X$ be a compact Kähler manifold of (complex) dimension $n$. Consider the complex of $\mathbb{C}$-vector spaces

$$
C^{\cdot}=\Omega_{X} \otimes \mathbb{C}\left[U, U^{-1}\right] \otimes \mathbb{C}\left[\hbar, \hbar^{-1}\right],
$$

where $\Omega_{X}=\oplus_{p, q} \Omega_{X}^{p, q}$ is the complex of global sections of the sheaves of $(p, q)$-forms on $X, \hbar$ and $U$ are formal independent variables, with $U$ of degree two. Our choice of notation wants to be suggestive of [15, in view of the analogies illustrated in the last section of this paper. On $C$ we consider the total differential $\delta_{C}=d_{C}^{\prime}+d_{C}^{\prime \prime}$, where $d_{C}^{\prime}=\hbar d$, with $d=\partial+\bar{\partial}$ the usual de Rham differential and

\footnotetext{
‡Partially supported by NSERC grants 72016789, 72024520 .

${ }^{\dagger}$ Partially supported by Humboldt Foundation Sofja Kovalevskaja Award.
} 
$d_{C}^{\prime \prime}=\sqrt{-1}(\bar{\partial}-\partial)$. The hypercohomology $\mathbb{H}^{\cdot}\left(C^{\cdot}, \delta_{C}\right)$ is then simply given by the infinite dimensional vector space $H^{\cdot}(X ; \mathbb{C}) \otimes \mathbb{C}\left[U, U^{-1}\right] \otimes \mathbb{C}\left[\hbar, \hbar^{-1}\right]$.

We also consider the positive definite inner product

$$
\left\langle\alpha \otimes U^{r} \otimes \hbar^{k}, \eta \otimes U^{s} \otimes \hbar^{t}\right\rangle:=\langle\alpha, \eta\rangle \delta_{r, s} \delta_{k, t},
$$

where $\langle\alpha, \eta\rangle$ denotes the Hodge inner product on forms $\Omega_{X}$, given by

$$
\langle\alpha, \eta\rangle:=\int_{X} \alpha \wedge * C(\bar{\eta}),
$$

with $C(\eta)=(\sqrt{-1})^{p-q}$, for $\eta \in \Omega_{X}^{p, q}$, and $\delta_{a, b}$ the Kronecker delta.

We then introduce certain cutoffs on $C$, which will allow us to recover the complex at arithmetic infinity of 9] from $C$.

To fix notation, for fixed $p, q \in \mathbb{Z}_{\geq 0}$ with $m=p+q$, let

$$
\lambda(q, r):=\max \{0,2 r+m, r+q\},
$$

where $2 r+m$ is the total degree of the complex. Let $\tilde{\Lambda}_{p, q} \subset \mathbb{Z}^{2}$ be the set of lattice points satisfying

$$
\tilde{\Lambda}_{q}=\left\{(r, k) \in \mathbb{Z}^{2}: k \geq \lambda(q, r)\right\},
$$

for $\lambda(q, r)$ as in (2.4).

For fixed $(p, q)$ with $m=p+q$, let $\mathfrak{C}_{p, q}^{m, 2 r} \subset C$ be the complex linear subspace given by the span of the elements of the form

$$
\alpha \otimes U^{r} \otimes \hbar^{k}
$$

where $\alpha \in \Omega_{X}^{p, q}$ and $(r, k) \in \tilde{\Lambda}_{q}$. We regard $\mathfrak{C}_{p, q}^{m, *}$ as a $2 \mathbb{Z}$-graded complex vector space.

Let $\mathfrak{C}$ be the direct sum of all the $\mathfrak{C}_{p, q}^{m, *}$, for varying $(p, q)$. We regard it as a $\mathbb{Z}$-graded complex vector space with total degree $2 r+m$.

In the cutoff (2.4), while the integer $2 r+m$ is just the total degree in $\Omega_{X} \otimes \mathbb{C}\left[U, U^{-1}\right]$, the constraint $k \geq r+q$ can be explained in terms of the Hodge filtration.

Let $\gamma^{\cdot}=F^{\cdot} \cap \bar{F}^{\cdot}$, where $F^{\cdot}$ and $\bar{F}^{\cdot}$ are the Hodge filtrations

$$
\begin{gathered}
F^{p} \Omega_{X}^{m}:=\bigoplus_{\substack{p^{\prime}+q=m \\
p^{\prime} \geq p}} \Omega_{X}^{p^{\prime}, q}, \\
\bar{F}^{q} \Omega_{X}^{m}:=\bigoplus_{\substack{p+q^{\prime \prime}=m \\
q^{\prime \prime} \geq q}} \Omega_{X}^{p, q^{\prime \prime}} .
\end{gathered}
$$

The condition defining $\mathfrak{C}$ can be rephrased in the following way.

Lemma 2.1. The complex $\mathfrak{C}$ has an equivalent description as $\mathfrak{C}^{i}=\bigoplus_{i=m+2 r} \mathfrak{C}^{m, 2 r}$, with

$$
\mathfrak{C}^{m, 2 r}=\bigoplus_{\substack{p+q=m \\ k \geq \max \{0,2 r+m\}}}\left(F^{m+r-k} \Omega_{X}^{m}\right) \otimes U^{r} \otimes \hbar^{k} .
$$

with the filtration $F^{\cdot}$ as in (2.7).

Proof. This follows immediately by

$$
F^{m+r-k} \Omega_{X}^{m}=\bigoplus_{\substack{p+q=m \\ k \geq r+q}} \Omega_{X}^{p, q}
$$


Let $c$ denote the complex conjugation operator acting on complex differential forms. We set $\mathcal{T}:=$ $\left(\mathfrak{C}^{-}\right)^{c=i d}$. This is the real complex

$$
\left(C^{\cdot}\right)^{c=i d}=\Omega_{X, \mathbb{R}} \otimes \mathbb{R}\left[U, U^{-1}\right] \otimes \mathbb{R}\left[\hbar, \hbar^{-1}\right] .
$$

Here $\Omega_{X, \mathbb{R}}^{m}$ is the $\mathbb{R}$-vector space of real differential forms of degree $m$, spanned by forms $\alpha=\xi+\bar{\xi}$, with $\xi \in \Omega_{X}^{p, q}$ and such that $p+q=m$, namely

$$
\Omega_{X, \mathbb{R}}^{m}=\bigoplus_{p+q=m}\left(\Omega_{X}^{p, q}+\Omega_{X}^{q, p}\right) .
$$

We have then the following equivalent description of $\mathcal{T}$.

Lemma 2.2. The complex $\mathcal{T}=\left(\mathfrak{C}^{\cdot}\right)^{c=i d}$ has the equivalent description $\mathcal{T}^{i}=\bigoplus_{i=m+2 r} \mathcal{T}^{m, 2 r}$ with

$$
\mathcal{T}^{m, 2 r}=\bigoplus_{\substack{p+q=m \\ k \geq \max \{0,2 r+m\}}}\left(\gamma^{m+r-k} \Omega_{X}^{m}\right) \otimes U^{r} \otimes \hbar^{k},
$$

with the filtration $\gamma^{\cdot}=F^{\cdot} \cap \bar{F}^{\cdot}$.

Proof. We have

$$
\begin{aligned}
F^{m+r-k} \Omega_{X}^{m} & =\bigoplus_{\substack{p+q=m \\
k \geq r+q}} \Omega_{X}^{p, q} \\
\bar{F}^{m+r-k} \Omega_{X}^{m} & =\bigoplus_{\substack{p+q=m \\
k \geq r+p}} \Omega_{X}^{p, q},
\end{aligned}
$$

hence one obtains

$$
\gamma^{m+r-k} \Omega_{X}^{m}=\bigoplus_{\substack{p+q=m \\ k \geq \frac{|p-q|+2 r+m}{2}}} \Omega_{X}^{p, q}
$$

where $(|p-q|+2 r+m) / 2=r+\max \{p, q\}$.

Notice that the inner product (2.3) is real valued on real forms, hence it induces an inner product on $\mathcal{T}$.

Lemma 2.2 suggests the following convenient description of $\mathcal{T}$; which we shall use in the following. For fixed $(p, q)$ with $m=p+q$, let

$$
\kappa(p, q, r):=\max \left\{0,2 r+m, \frac{|p-q|+2 r+m}{2}\right\} .
$$

Then let $\Lambda_{p, q} \subset \mathbb{Z}^{2}$ be the set

$$
\Lambda_{p, q}=\left\{(r, k) \in \mathbb{Z}^{2}: k \geq \kappa(p, q, r)\right\}
$$

with $\kappa(p, q, r)$ as in (2.13).

Proposition 2.3. The elements of the $\mathbb{Z}$-graded real vector space $\mathcal{T}$ are linear combinations of elements of the form

$$
\alpha \otimes U^{r} \otimes \hbar^{k}
$$

with $\alpha \in\left(\Omega_{X}^{p, q}+\Omega_{X}^{q, p}\right), \alpha=\xi+\bar{\xi}$, for some $(p, q)$ with $p+q=m$, and $(r, k)$ in the corresponding $\Lambda_{p, q}$. 


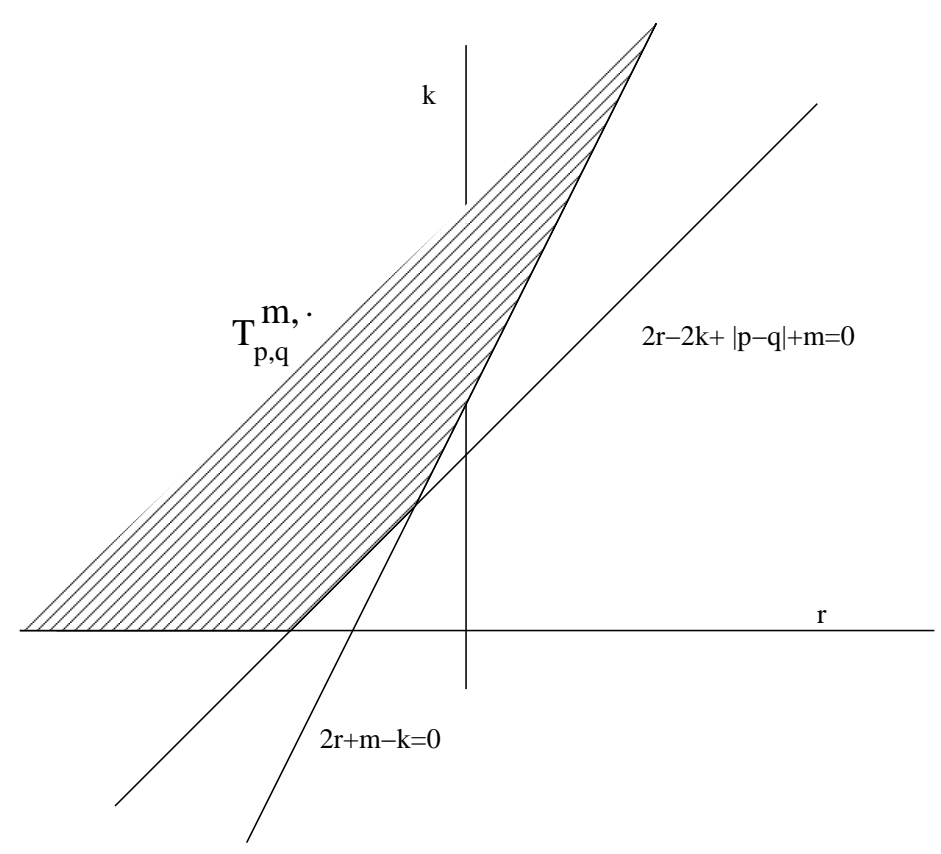

FiguRE 1. The region $\Lambda_{p, q} \subset \mathbb{Z}^{2}$ defining $\mathcal{T}_{p, q}^{m,}$

Proof. By Lemma 2.1] we have seen that the cutoff $\lambda(q, r)$ of (2.4) corresponds to the Hodge filtration $F^{*}$, while Lemma 2.2 shows that, when we impose $c=i d$ we can describe $\left(\mathfrak{C}^{-}\right)^{c=i d}$ in terms of the $\gamma$-filtration as in (2.12). For fixed $(p, q)$ with $p+q=m$, this corresponds to the fact that

$$
\mathcal{T}_{p, q}^{m, 2 r}:=\left(\mathfrak{C}_{p, q}^{m, 2 r} \oplus \mathfrak{C}_{q, p}^{m, 2 r}\right)^{c=i d}
$$

is the real vector space generated by elements of the form (2.15), where $\alpha=\xi+\bar{\xi} \in\left(\Omega_{X}^{p, q}+\Omega_{X}^{q, p}\right)$ is a real form and the indices $(r, k)$ satisfy the conditions $k \geq 0, k \geq 2 r+m$ and $k \geq r+\max \{p, q\}$. Equivalently, $(r, k) \in \Lambda_{p, q}$. Notice that, since $\kappa(p, q, r)=\kappa(q, p, r)$, we have $\Lambda_{p, q}=\Lambda_{q, p}$. In fact $\Lambda_{p, q}$ depends on $(p, q)$ only through $|p-q|$ and $m=p+q$.

Figure 1 describes, for fixed values of $m$ and $|p-q|$, the effect of the cutoff 2.13) on the varying indices $(r, k)$. Namely, for fixed $(p, q)$ with $p+q=m$, the region $\Lambda_{p, q} \subset \mathbb{Z}^{2}$ defined in (2.14) is the shaded region in Figure 1. The graph of the function $k=\kappa(p, q, r)$ of (2.13) is the boundary of the shaded region in the Figure.

By construction, the real vector space $\mathcal{T}_{p, q}^{m, 2 r}$ is the linear span of the (2.15) with $(r, k) \in \Lambda_{p, q}$ and $\mathcal{T}$. is the direct sum of all the $\mathcal{T}_{p, q}^{m, *}$, for varying $(p, q)$, viewed as a $\mathbb{Z}$-graded real vector space with total degree $2 r+m$. Namely, we can think of a single $\mathcal{T}_{p, q}^{m, *}$ as a "slice" of $\mathcal{T}$. for fixed $(p, q)$, namely, for each $(p, q)$ there is a corresponding Figure 1 and $\mathcal{T}$ ' is obtained when considering the union of all of them.

The differentials $d_{C}^{\prime}$ and $d_{C}^{\prime \prime}$ induce corresponding differentials $d^{\prime}$ and $d^{\prime \prime}$ on $\mathcal{T}$; where $d^{\prime}=d_{C}^{\prime}=\hbar d$ and $d^{\prime \prime}=P^{\perp} d_{C}^{\prime \prime}$, with $P^{\perp}$ the orthogonal projection of $\left(C^{\cdot}\right)^{c=i d}$ onto $\mathcal{T}$. Notice that, since $d^{\prime}$ and $d^{\prime \prime}$ change the values of $(p, q)$, the differentials move from one "slice" $\mathcal{T}_{p, q}^{m, *} \subset \mathcal{T}$ ' to another.

2.1. Operators. In the formulation introduced above, we then obtain the very simple description of the operators $N$ and $\Phi$ of $[9]$ as

$$
N=U \hbar \quad \Phi=-U \frac{\partial}{\partial U} .
$$


In 9] these represent, respectively, a logarithm of the local monodromy and a logarithm of Frobenius at arithmetic infinity. We consider the Hilbert space completion of $\mathcal{T}$ in the inner product induced by (2.2). With a slight abuse of notation, we still denote this Hilbert space by $\mathcal{T}$. The linear operator $N$ satisfies $N^{*} N=P_{1}$ and $N N^{*}=P_{2}$, where $P_{1}$ and $P_{2}$ denote, respectively, the orthogonal projections onto the closed subspaces $\operatorname{Ker}(N)^{\perp}$ and $\operatorname{Coker}(N)^{\perp}$ of $\mathcal{T}$. The operator $N$ also has the property that $\left[N, d^{\prime}\right]=\left[N, d^{\prime \prime}\right]=0$. The operator $\Phi$ is an unbounded, self adjoint operator with spectrum $\operatorname{Spec}(\Phi)=\mathbb{Z}$. It also satisfies $\left[\Phi, d^{\prime}\right]=\left[\Phi, d^{\prime \prime}\right]=0$.

Notice that, unlike the differentials $d^{\prime}$ and $d^{\prime \prime}$ that move between different slices $\mathcal{T}_{p, q}^{m, *}$ of $\mathcal{T}$, the monodromy map $N$ does not change the values $(p, q)$.

This means that, in addition to the result of Corollary 4.4 of [9] on the "global" properties of injectivity and surjectivity of the map $N: \mathcal{T} \cdot \mathcal{T}^{\cdot+2}$, we can also give an analogous "local" result describing the properties of the map $N: \mathcal{T}_{p, q}^{m, *} \rightarrow \mathcal{T}_{p, q}^{m, *+2}$, restricted to an action on a fixed "slice" (i.e. for fixed $p$ and $q$ ). In this case, we obtain the following result.

Proposition 2.4. The endomorphism $N: \mathcal{T}_{p, q}^{m, 2 r} \rightarrow \mathcal{T}_{p, q}^{m, 2(r+1)}$ has the following properties:

(1) $N$ is surjective iff $r$ is in the range $r>-\max \{p, q\}$

(2) $N$ is injective iff $r$ is in the range $r<-\min \{p, q\}$.

Proof. (1) For fixed $(p, q)$ with $p+q=m$, let $\Lambda_{p, q} \subset \mathbb{Z}^{2}$ denote the shaded region in Figure 1 as in (2.14). Let $Z_{p, q} \subset \mathbb{Z}^{2}$ denote the set of lattice points $Z_{p, q}=\left\{(r, k) \in \mathbb{Z}^{2}: r>-\max \{p, q\}\right\}$.

The point $(-\max \{p, q\}, 0) \in \Lambda_{p, q}$ is the intersection point of the lines $k=0$ and $2 r-2 k+m+|p-q|=0$ in the boundary of $\Lambda_{p, q}$. Thus, one sees that the only points in $(r, k) \in \Lambda_{p, q}$ such that $(r-1, k-1) \notin \Lambda_{p, q}$ are those of the form $(r, 0)$ with $r \leq-\max \{p, q\}$. This shows that every point $(r, k) \in \Lambda_{p, q} \cap Z_{p, q}$ has the property that $(r-1, k-1) \in \Lambda_{p, q}$, hence $N$ is surjective in the range $r>-\max \{p, q\}$. It also shows that, for every $r \leq-\max \{p, q\}$, the point $(r, 0)$ in $\Lambda_{p, q}$ is such that $(r-1,-1) \notin \Lambda_{p, q}$, so that $\mathrm{N}$ cannot be surjective in the range $r \leq-\max \{p, q\}$.

(2) The case of injectivity is proved similarly. Let

$$
W_{p, q}=\left\{(r, k) \in \mathbb{Z}^{2}: r<-\min \{p, q\}\right\} .
$$

Notice that the only points $(r, k) \in \Lambda_{p, q}$ such that $(r+1, k+1) \notin \Lambda_{p, q}$ are those on the boundary line $k=2 r+m$. The point $(-\min \{p, q\},|p-q|)$ is the intersection point of the lines $k=2 r+m$ and $2 r-2 k+m+|p-q|=0$ in the boundary of $\Lambda_{p, q}$. Thus, we see that every point $(r, k) \in \Lambda_{p, q} \cap W_{p, q}$ is such that $(r+1, k+1) \in \Lambda_{p, q}$, and conversely, for all $r \geq-\min \{p, q\}$ there exists a point $(r, k=$ $2 r+m) \in \Lambda_{p, q}$ such that $(r+1, k+1) \notin \Lambda_{p, q}$, hence $N$ is injective in the range $r<-\min \{p, q\}$, while it cannot be injective for $r \geq-\min \{p, q\}$.

The complex $(\mathcal{T} ; \delta)$ has another important structure, given by the Lefschetz operator, which, together with the polarization and the monodromy, endows $(\mathcal{T} \cdot \delta)$ with the structure of a polarized HodgeLefschetz module, in the sense of Deligne and Saito ( $c f$. 9], 18, 24]). The Lefschetz endomorphism $\mathbb{L}$ is given by

$$
\mathbb{L}=(\cdot \wedge \omega) U^{-1}
$$

where $\omega$ is the canonical real closed $(1,1)$-form determined by the Kähler structure. The Lefschetz operator satisfies $\left[\mathbb{L}, d^{\prime}\right]=\left[\mathbb{L}, d^{\prime \prime}\right]=0$.

Notice that, unlike the monodromy operator $N$ that preserves the "slices" $\mathcal{T}_{p, q}^{m, *}$, the Lefschetz moves between different slices, namely

$$
\mathbb{L}: \mathcal{T}_{p, q}^{m, *} \rightarrow \mathcal{T}_{p+1, q+1}^{m+2, *-1}
$$




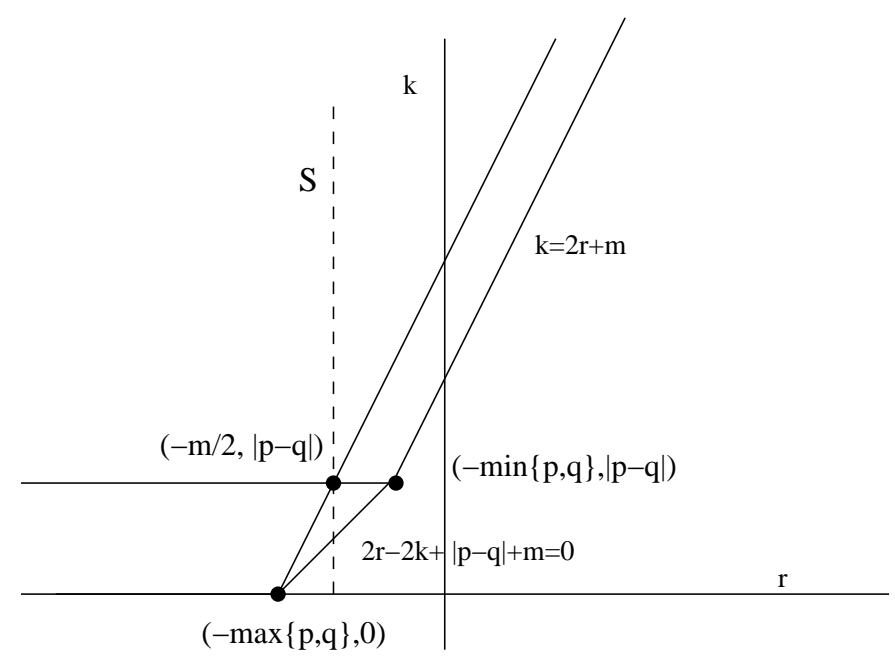

Figure 2. The duality $S$

2.2. Dualities. There are two important duality maps on the complex $\mathcal{T}$. The first is defined on forms by

$$
S: \alpha \otimes U^{r} \otimes \hbar^{2 r+m+\ell} \mapsto \alpha \otimes U^{-(r+m)} \otimes \hbar^{\ell},
$$

for $\alpha \in \Omega_{X}^{m}$, and it induces, at the level of cohomology, the duality map of Proposition 4.8 of [9]. The map $S$ induces, in particular, the duality between kernel and cokernel of the monodromy map in cohomology, described in [9] and [10] in terms of powers of the monodromy ( $c f$. Proposition [2.5] below).

The other duality is given by the map

$$
\tilde{S}: \alpha \otimes U^{r} \otimes \hbar^{k} \mapsto C(* \alpha) \otimes U^{r-(n-m)} \otimes \hbar^{k} .
$$

Proposition 2.5. Let $S$ and $\tilde{S}$ be the maps defined in (2.19) and (2.20).

(1) The map $S: \mathcal{T} \rightarrow \mathcal{T}$ is an involution, namely $S^{2}=1$. It gives a collection of linear isomorphisms

$$
S=N^{-(2 r+m)}: \operatorname{span}\left\{\alpha \otimes U^{r} \otimes \hbar^{2 r+m+\ell}\right\} \rightarrow \operatorname{span}\left\{\alpha \otimes U^{-(r+m)} \otimes \hbar^{\ell}\right\},
$$

realized by powers $N^{-(2 r+m)}$ of the monodromy.

(2) The map $\tilde{S}: \mathcal{T} \rightarrow \mathcal{T}$ is an involution, $\tilde{S}^{2}=1$. The map induced by $\tilde{S}$ on the primitive part of the cohomology, with respect to the Lefschetz decomposition, agrees (up to a non-zero real constant) with the power $\mathbb{L}^{n-m}$ of the Lefschetz operator.

Proof. (1) The result for $S$ follows directly from the definition, in fact, we have

$$
\begin{gathered}
S^{2}\left(\alpha \otimes U^{r} \otimes \hbar^{2 r+m+\ell}\right)=S\left(\alpha \otimes U^{-(r+m)} \otimes \hbar^{\ell}\right) \\
=\alpha \otimes U^{r+m-m} \otimes \hbar^{\ell+2(r+m)-m}=\alpha \otimes U^{r} \otimes \hbar^{2 r+m+\ell} .
\end{gathered}
$$

This means that the duality $S$ preserve the "slices" $\mathcal{T}_{p, q}^{m, *}$ and on them it can be identified with the symmetry of $\mathcal{T}$. obtained by reflection of the shaded area of Figure 1 along the line illustrated in Figure 2] The elements of the form $\alpha \otimes U^{-m / 2} \otimes \hbar^{|p-q|}$, for $\alpha \in \Omega_{X}^{m}$ are fixed by the involution $S$ since

$$
S\left(\alpha \otimes U^{-m / 2} \otimes \hbar^{|p-q|}\right)=\alpha \otimes U^{\frac{m}{2}-m} \otimes \hbar^{|p-q|+\frac{2 m}{2}-m}=\alpha \otimes U^{-m / 2} \otimes \hbar^{|p-q|} .
$$


We prove (2). The map $\tilde{S}$ preserves $\mathcal{T}$, since the cutoffs described by the conditions $k \geq 0,2 r+m-$ $2 k+|p-q| \leq 0$ and $k \geq 2 r+m$ are preserved by mapping $r \mapsto r-(n-m), k \mapsto k, m \mapsto 2 n-m$, $(p, q) \mapsto(n-q, n-p)$. We have $\tilde{S}^{2}=1$, since

$$
\begin{gathered}
\tilde{S}^{2}\left(\alpha \otimes U^{r} \otimes \hbar^{k}\right)=(\sqrt{-1})^{p-q} \tilde{S}\left(* \alpha \otimes U^{r-(n-m)} \otimes \hbar^{k}\right) \\
=(\sqrt{-1})^{p-q}(\sqrt{-1})^{n-q-(n-p)} *^{2} \alpha \otimes U^{r-(n-m)-(n-(2 n-m))} \otimes \hbar^{k} \\
=(\sqrt{-1})^{2(p-q)}(-1)^{m} \alpha \otimes U^{r} \otimes \hbar^{k},
\end{gathered}
$$

where we used $*^{2}=(-1)^{m(2 n-m)}=(-1)^{m}$.

Let $P^{m}(X)$ be the primitive part of the cohomology $H^{m}(X, \mathbb{C})$, with respect to the Lefschetz decomposition ( $c f .[29] \S \mathrm{V} .6)$. Let $J$ be the operator induced on $P^{p, q}(X)$ by $C(\eta)=(\sqrt{-1})^{p-q} \eta$. On the primitive cohomology one has the identification (up to multiplication by a non-zero real constant)

$$
\mathbb{L}^{n-m} J \eta=* \eta \text {. }
$$

In particular, (2.21) implies that the map $\tilde{S}$ agrees (up to a normalization factor) with $\mathbb{L}^{n-m}$, on the primitive cohomology.

Thus, we can think of the two dualities $S$ and $\tilde{S}$ as related, respectively, to the action of $N^{2 r+m}$ and $\mathbb{L}^{n-m}$, i.e. of powers of the monodromy and Lefschetz.

2.3. Representations. The nilpotent endomorphisms $\mathbb{L}$ and $N$ of $\mathcal{T}$ introduced above define two representations of $\operatorname{SL}(2, \mathbb{R})$ on $\mathcal{T}$ as follows. With the notation

$$
\chi(\lambda):=\left(\begin{array}{cc}
\lambda & 0 \\
0 & \lambda^{-1}
\end{array}\right) \lambda \in \mathbb{R}^{*}, \quad u(s):=\left(\begin{array}{cc}
1 & s \\
0 & 1
\end{array}\right) s \in \mathbb{R}, \quad w:=\left(\begin{array}{cc}
0 & 1 \\
-1 & 0
\end{array}\right)
$$

we define $\sigma^{L}$ and $\sigma^{R}$ by

$$
\begin{gathered}
\sigma^{L}(\chi(\lambda))=\lambda^{-n+m}, \quad \sigma^{L}(u(s))=\exp (s \mathbb{L}), \quad \sigma^{L}(w)=(\sqrt{-1})^{n} C \tilde{S} . \\
\sigma^{R}(\chi(\lambda))=\lambda^{2 r+m}, \quad \sigma^{R}(u(s))=\exp (s N), \quad \sigma^{R}(w)=C S .
\end{gathered}
$$

Here $C$ is the operator on forms $C(\eta)=(\sqrt{-1})^{p-q}$ for $\eta \in \Omega_{X}^{p, q}$, and $S$ and $\tilde{S}$ the dualities on $\mathcal{T}$, as in Proposition 2.5. The results of [18], 9] and [10] yield the following.

Proposition 2.6. The operators (2.23) and (2.24) on $\mathcal{T}$ define a representation $\sigma=\left(\sigma^{L}, \sigma^{R}\right)$ : $\mathrm{SL}(2, \mathbb{R}) \times \mathrm{SL}(2, \mathbb{R}) \rightarrow \operatorname{Aut}\left(\mathcal{T}^{\cdot}\right)$. In the representation $\sigma^{L}$, the group $\mathrm{SL}(2, \mathbb{R})$ acts by bounded operators on the completion of $\mathcal{T}$ in the inner product (2.2). Both $\mathrm{SL}(2, \mathbb{R})$ actions commute with the Laplacian $\square=\delta \delta^{*}+\delta^{*} \delta$, hence they define induced representations on the cohomology $\mathbb{H}^{\cdot}(\mathcal{T} \cdot \delta)$.

Proof. For completeness, we give here a simple proof of the proposition. In order to show that we have representations of $\mathrm{SL}(2, \mathbb{R})$ it is sufficient ([19] $\S \mathrm{XI} .2)$ to check that (2.23) and (2.24) satisfy the relations

$$
\begin{aligned}
\sigma(w)^{2} & =\sigma(\chi(-1)) \\
\sigma(\chi(\lambda)) \sigma(u(s)) \sigma\left(\chi\left(\lambda^{-1}\right)\right) & =\sigma\left(u\left(s \lambda^{2}\right)\right)
\end{aligned}
$$

We show it first for $\sigma^{R}$. We show that we have $\sigma^{R}(w)=(-1)^{m}$, as in 10. This follows directly from the fact that $S^{2}=1$, since $\sigma^{R}(w)^{2}=C S C S=(\sqrt{-1})^{2(p-q)}=(-1)^{m}$. Thus, we have $\sigma^{R}(w)^{2}=$ $(-1)^{m}=\left.\lambda^{2 r+m}\right|_{\lambda=-1}$ and the first relation of $(2.25)$ is satisfied. To check the second relation notice that, on an element $\alpha \otimes U^{r} \otimes \hbar^{k}$ with $\alpha \in \Omega_{X}^{m}$ we have

$$
\begin{gathered}
\sigma^{R}(\chi(\lambda)) \sigma^{R}(u(s)) \sigma^{R}\left(\chi\left(\lambda^{-1}\right)\right) \alpha \otimes U^{r} \otimes \hbar^{k}= \\
\sigma^{R}(\chi(\lambda))\left(1+s N+\frac{s^{2}}{2} N^{2}+\cdots\right) \lambda^{-(2 r+m)} \alpha \otimes U^{r} \otimes \hbar^{k}=
\end{gathered}
$$




$$
\left(1+\lambda^{2(r+1)+m} s N \lambda^{-(2 r+m)}+\lambda^{2(r+2)+m} \frac{s^{2}}{2} N^{2} \lambda^{-(2 r+m)}+\cdots\right) \alpha \otimes U^{r} \otimes \hbar^{k}=\exp \left(s \lambda^{2} N\right) \alpha \otimes U^{r} \otimes \hbar^{k},
$$

hence the second relation is satisfied.

We show that $\sigma^{L}$ also satisfies the relations (2.25). Again, we first show that $\sigma^{L}(w)=(-1)^{n+m}$, as in [9]. We have

$$
C \tilde{S}\left(\alpha \otimes U^{r} \otimes \hbar^{k}\right)=(\sqrt{-1})^{n-q-n+p}(\sqrt{-1})^{p-q} * \alpha \otimes U^{r-(n-m)} \otimes \hbar^{k},
$$

hence

$$
\begin{gathered}
\sigma^{L}(w)^{2}\left(\alpha \otimes U^{r} \otimes \hbar^{k}\right)=(-1)^{n}(-1)^{m} C \tilde{S}\left(* \alpha \otimes U^{r-(n-m)} \otimes \hbar^{k}\right) \\
=(-1)^{n}(-1)^{m}(-1)^{m}(\sqrt{-1})^{p-q}(\sqrt{-1})^{n-q-n+p} \alpha \otimes U^{r} \otimes \hbar^{k}=(-1)^{n}(-1)^{m} \alpha \otimes U^{r} \otimes \hbar^{k}
\end{gathered}
$$

where, in the left-hand side, we used $*^{2}=(-1)^{m}$.

Thus, we have $\sigma^{L}(w)^{2}=(-1)^{n+m}=\left.\lambda^{m-n}\right|_{\lambda=-1}$. Moreover, we have

$$
\begin{gathered}
\sigma^{L}(\chi(\lambda)) \sigma^{L}(u(s)) \sigma^{L}\left(\chi\left(\lambda^{-1}\right)\right) \alpha \otimes U^{r} \otimes \hbar^{k}= \\
\sigma^{L}(\chi(\lambda))\left(1+s \mathbb{L}+\frac{s^{2}}{2} \mathbb{L}^{2}+\cdots\right) \lambda^{n-m} \alpha \otimes U^{r} \otimes \hbar^{k}= \\
\left(1+\lambda^{-n+m+2} s \mathbb{L} \lambda^{n-m}+\lambda^{-n+m+4} \frac{s^{2}}{2} \mathbb{L}^{2} \lambda^{n-m}+\cdots\right) \alpha \otimes U^{r} \otimes \hbar^{k}=\exp \left(s \lambda^{2} \mathbb{L}\right) \alpha \otimes U^{r} \otimes \hbar^{k},
\end{gathered}
$$

hence the second relation is also satisfied.

The fact that $\sigma^{L}(\chi(\lambda))$ is a bounded operator in the inner product induced by (2.2), while for $\lambda \neq \pm 1$ the operators $\sigma^{R}(\chi(\lambda))$ are unbounded is clear from the fact that the index $2 r+m$ ranges over all of $\mathbb{Z}$, while $-n \leq m-n \leq n$. For the fact that $\left[\square, \sigma^{L}\right]=\left[\square, \sigma^{R}\right]=0$ we refer to $[9]$.

2.4. Ring of differential operators. Let $\mathcal{D}$ denote the algebra of differential operators on a 1dimensional complex torus $T_{\mathbb{C}}$, generated by the operators $Q=e^{z}$ and $P=\frac{\partial}{\partial z}$ satisfying the commutation relation

$$
P Q-Q P=Q
$$

Let $\mathcal{R}$ be the ring of functions defining the coefficients of the differential operators in $\mathcal{D}$. This is a subring of the ring of functions on $\mathbb{C}^{*}$. For $\mathcal{R}=\mathbb{C}[Q]$ we obtain $\mathcal{D}=\mathbb{C}[P, Q] /(P Q-Q P=Q)$.

Since the operators $N$ and $\Phi$ satisfy the commutation relation $[\Phi, N]=-N$ and the operators $\mathbb{L}$ and $\Phi$ satisfy the commutation relation $[\Phi, \mathbb{L}]=\mathbb{L}$, the pairs of operators $(N,-\Phi)$ and $(\mathbb{L}, \Phi)$ define actions $\pi^{R}$ and $\pi^{L}$ of $\mathcal{D}$ on the complex $\mathfrak{C}^{-}$and on its cohomology, by setting

$$
\begin{array}{ll}
\pi^{L}(P)=\Phi & \pi^{L}(Q)=\mathbb{L} \\
\pi^{R}(P)=-\Phi & \pi^{R}(Q)=N .
\end{array}
$$

There is an induced action of the ring $\mathcal{D}=\mathbb{R}[P, Q] /(P Q-Q P=Q)$ on the complex $\mathcal{T}$. and on its cohomology. 
2.5. Weil-Deligne group at arithmetic infinity. On $\mathcal{T}$. consider the "Frobenius flow"

$$
F_{t}=e^{t \Phi}, \quad \forall t \in \mathbb{R}
$$

generated by the operator $\Phi$. We write $F=F_{1}$. This satisfies

$$
F N F^{-1}=e^{-1} N \text {. }
$$

Thus, the operators $F$ and $N$ can be thought of as defining an analog at arithmetic infinity of the Weil-Deligne group $\mathbb{G}_{a} \rtimes W_{K}$, which acts on the finite dimensional vector space associated to the étale cohomology of the geometric generic fiber of a local geometric degeneration for $K$ a non-archimedean local field. In fact, in that case, the action of the Frobenius $\varphi \in W_{K}$ on $\mathbb{G}_{a}$ is given by

$$
\varphi x \varphi^{-1}=q^{-1} x
$$

where $q$ is the cardinality of the residue field. The formal replacement of $q$ by $e$ and of $\varphi$ by $F$ determines (2.29) from (2.30). In the archimedean case, this "Weil-Deligne group" acts directly at the level of the complex, not just on the cohomology.

\section{Archimedean COHOMOLOGY}

We now describe the relation between the complex $\left(\mathcal{T} \cdot \delta=d^{\prime}+d^{\prime \prime}\right)$ defined in the first section and the cohomology theory at arithmetic infinity developed in 9 .

On a smooth projective algebraic variety $X$ of dimension $n$ over $\mathbb{C}$ or $\mathbb{R}$, the complex of Tate-twisted real differential forms introduced in (4.1) of [9] is defined as

$$
K^{i, j, k}= \begin{cases}\bigoplus_{\substack{p+q=j+n \\|p-q| \leq 2 k-i}}\left(\Omega_{X}^{p, q} \oplus \Omega_{X}^{q, p}\right)_{\mathbb{R}} \otimes_{\mathbb{R}} \mathbb{R}\left(\frac{n+j-i}{2}\right) & \text { if } j+n-i \equiv 0(2), k \geq \max (0, i) \\ 0 & \text { otherwise, }\end{cases}
$$

for $i, j, k \in \mathbb{Z}$. Here $\mathbb{R}(r)$ denotes the real Hodge structure $\mathbb{R}(r):=(2 \pi \sqrt{-1})^{r} \mathbb{R}$, and the differentials $d^{\prime}$ and $d^{\prime \prime}$ are given by

$$
\begin{gathered}
d^{\prime}: K^{i, j, k} \rightarrow K^{i+1, j+1, k+1}, \quad d^{\prime}(\alpha)=d(\alpha) \\
d^{\prime \prime}: K^{i, j, k} \rightarrow K^{i+1, j+1, k}, \quad d^{\prime \prime}(\alpha)=\sqrt{-1}(\bar{\partial}-\partial)(\alpha) \quad\left(\text { projected onto } K^{i+1, j+1, k}\right) .
\end{gathered}
$$

The inner product on $\mathbb{H}^{\cdot}\left(K^{\prime}, d^{\prime}+d^{\prime \prime}\right)$ is defined in terms of the bilinear form

$$
Q(\alpha, \eta)=\int_{X} \mathbb{L}^{n-m} \alpha \wedge J \bar{\eta},
$$

for $\eta, \alpha$ in the primitive part $P^{m}(X)$ of the de Rham cohomology $H^{m}(X, \mathbb{R})$ with respect to the Lefschetz decomposition.

The relation between the complex $\mathcal{T}$. and the total complex $K^{\cdot}$ of (3.1) is described by the following result, which shows that the complex $\mathcal{T}$ is identified with $K^{\cdot}$ after applying the simple change of variables

$$
i=m+2 r, \quad r=-\frac{n+j-i}{2}, \quad j=-n+m
$$

to the indices of $\mathcal{T}$, taking fixed points under complex conjugation $(c=i d)$ and replacing the variable $U$ by a Tate twist.

Proposition 3.1. Upon identifying the formal variable $U^{-1}$ with the Tate twist given by multiplication by $2 \pi \sqrt{-1}$, we obtain an isomorphism of complexes

$$
\left(\left.\mathcal{T} \cdot\right|_{U=(2 \pi \sqrt{-1})^{-1}}, \delta\right) \cong\left(K^{\prime}, d^{\prime}+d^{\prime \prime}\right),
$$

given by a reparameterization of the indices. Up to a normalization factor, the inner product induced by (2.2) on the cohomology $\mathbb{H}^{\cdot}\left(\left.\mathcal{T} \cdot\right|_{U=(2 \pi \sqrt{-1})^{-1}}, \delta\right)$ agrees with the one defined by (3.4) on $\mathbb{H}^{\cdot}\left(K^{\prime}, d^{\prime}+d^{\prime \prime}\right)$. 
Proof. We define a homomorphism

$$
I: \mathcal{T} \rightarrow K \cdot
$$

as follows. For fixed $p, q$ with $p+q=m$, consider the region $\Lambda_{p, q} \subset \mathbb{Z}^{2}$ as in (2.14), with $\kappa(p, q, r)$ as in 2.13). For every $\alpha \in\left(\Omega_{X}^{p, q} \oplus \Omega_{X}^{q, p}\right) \mathbb{R}_{\mathbb{R}}$ a real form, $\alpha=\xi+\bar{\xi}$, with $\xi \in \Omega_{X}^{p, q}$, and for every point $(r, k) \in \Lambda_{p, q}$, we have

$$
\alpha \otimes U^{r} \otimes \hbar^{k} \in \mathcal{T}_{p, q}^{m, 2 r}
$$

and, by Proposition 2.3. every element of $\mathcal{T}$ is a linear combination of elements of this form, for varying $(p, q)$ and corresponding $(r, k) \in \Lambda_{p, q}$.

We now define the map $I$ in the following way. To an element

$$
(\xi+\bar{\xi}) \otimes U^{r} \otimes \hbar^{k}
$$

with $\xi \in \Omega_{X}^{p, q}$, with $p+q=m$, and with $k \geq \kappa(p, q, r)$, we assign an element $I(\eta) \in K^{i, j, k}$, with the same index $k$ and with

$$
i=m+2 r, \quad \text { and } \quad j=-n+m,
$$

by setting

$$
I(\eta)=(2 \pi \sqrt{-1})^{-r}(\xi+\bar{\xi}) .
$$

In fact, for $(i, j)$ as in (3.8), the index $r \in \mathbb{Z}$ can be written in the form

$$
r=-\frac{n+j-i}{2}, \quad \text { where } n+j-i=0 \quad \bmod 2 .
$$

Thus, the element (3.9) can be written as

$$
(2 \pi \sqrt{-1})^{\frac{n+j-i}{2}}(\xi+\bar{\xi}) .
$$

By the definition (3.1), to check that this is an element in $K^{i, j, k}$, it is sufficient to verify that $p+q=$ $j+n$, and that the conditions $|p-q| \leq 2 k-i$ and $k \geq \max \{0, i\}$ are satisfied. Since $j=-n+m$ and $p+q=m$ we have $p+q=j+n$. The index $k$ is the same as in (3.7), hence it satisfies $k \geq \kappa(p, q, r)$. This means that $k \geq 0$ and that $k \geq 2 r+m=i$, so that $k \geq \max \{0, i\}$ satisfied. Since $k \geq \kappa(p, q, r)$ also implies $k \geq(|p-q|+2 r+m) / 2$, which, by $i=2 r+m$ is the condition $|p-q| \leq 2 k-i$. It is clear that the map $I$ defined this way is injective.

Thus, we have shown that, for every real form $\alpha \in\left(\Omega_{X}^{p, q} \oplus \Omega_{X}^{q, p}\right)_{\mathbb{R}}$ and for every lattice point $(r, k) \in \Lambda_{p, q}$ we have a unique corresponding element in the complex $K^{i, j, k}$.

The map $I$ is also surjective, hence a linear isomorphism. In fact, every element in $K$ is a linear combination of elements of the form (3.10) in $K^{i, j, k}$, for $\xi \in \Omega_{X}^{p, q}$, and indices $(i, j, k) \in \mathbb{Z}^{3}$ satisfying $n+j-i=0 \bmod 2, p+q=j+n, k \geq \max \{i, 0\}$ and $|p-q| \leq 2 k-i$. It is sufficient to show that, for any such element, there exists a point $(r, k) \in \Lambda_{p, q}$ such that

$$
I\left((\xi+\bar{\xi}) \otimes U^{r} \otimes \hbar^{k}\right)=(2 \pi \sqrt{-1})^{\frac{n+j-i}{2}}(\xi+\bar{\xi}) \in K^{i, j, k} .
$$

This is achieved by taking the point

$$
\left(r=-\frac{n+j-i}{2}, k\right) .
$$

Since $n+j-i=0 \bmod 2$ this point is in $\mathbb{Z}^{2}$, and since under the change of variables (3.8) the conditions $k \geq \max \{i, 0\}$ and $|p-q| \leq 2 k-i$ are equivalent to the condition $k \geq \kappa(p, q, r)$ of (2.13), the point (3.11) is in $\Lambda_{p, q}$.

The map $I$ is compatible with the differentials, namely

$$
I(\delta \eta)=\left(d^{\prime}+d^{\prime \prime}\right) I(\eta),
$$

where on the left hand side $\delta=d_{C}^{\prime}+P^{\perp} d_{C}^{\prime \prime}$ is the differential on $\mathcal{T}$ and on the right hand side $d^{\prime}+d^{\prime \prime}$ is as in (3.2) (3.3). To see this, first notice that the differential $d^{\prime}$ of (3.2) satisfies

$$
d^{\prime}=I d_{C}^{\prime} I^{-1}=I \hbar d I^{-1}: K^{2 r+m,-n+m, k} \rightarrow K^{2 r+(m+1),-n+(m+1), k+1} .
$$


The analogous statement $d^{\prime \prime}=I P^{\perp} d_{C}^{\prime \prime} I^{-1}$ for the differential $d^{\prime \prime}$ of (3.3) also involves the fact that the orthogonal projection onto $K^{i+1, j+1, k}$ in (3.3), induced by the inner product (3.4) agrees with the corresponding orthogonal projection $P^{\perp}$ in $\mathcal{T}$. induced by the inner product (2.2).

The identification (2.21) implies that the inner product (3.4) on $\mathbb{H}^{\cdot}\left(K^{\prime}, d^{\prime}+d^{\prime \prime}\right)$ considered in [9] and the inner product induced by (2.2) agree up to a normalization factor.

In particular, the 'weight type' condition $|a-b| \leq 2 k-i$ on the real forms in (3.1) describes, as in (2.7) (2.8), the filtration $\gamma^{\prime}:=F^{\cdot} \cap \bar{F}^{\cdot}$ on the complex of real differential forms on $X$. It follows that the complex $K^{i, j, k}$ has a real analytic type, even when $X$ defined over $\mathbb{C}$ does not have a real structure.

To the abelian group $K^{i, j, k}$ we assign the weight: $-n-j+i \in \mathbb{Z}$. Keeping in mind that $\mathbb{R}\left(\frac{n+j-i}{2}\right)$ is the real Hodge structure of rank one and pure bi-degree $\left(-\frac{n+j-i}{2},-\frac{n+j-i}{2}\right)$, we obtain the following description in terms of the filtration $\gamma^{\prime}$ :

$$
K^{i, j, k}=\gamma^{\frac{n+j+i}{2}-k} \Omega_{X}^{n+j} \otimes_{\mathbb{R}} \mathbb{R}\left(\frac{n+j-i}{2}\right)=\gamma^{\frac{n+j+i}{2}-k} \Omega_{X}^{n+j} \otimes_{\mathbb{R}} \gamma^{-\frac{n+j-i}{2}} \mathbb{R} .
$$

When considering the tensor product of the two structures one sees that the index of the $\gamma$-filtration on the product $\left(i . e\right.$. on $\left.K^{i, j, k}\right)$ is $i-k$.

3.1. Deligne cohomology. By Proposition 3.1 the complex $\mathcal{T}$. is related to the real Deligne cohomology $H_{\mathcal{D}}^{*}(X, \mathbb{R}(r))$. These groups can be computed via the Deligne complex $\left(C_{\mathcal{D}}^{*}(r), d_{\mathcal{D}}\right)(c f$. [3] , 9]). The relation of $\left(C_{\mathcal{D}}^{*}(r), d_{\mathcal{D}}\right)$ to the complex $\left(\mathcal{T}^{*}, \delta\right)$ is given by the following result of [9] (Prop. 4.1), which, for convenience, we reformulate here in our notation.

Proposition 3.2. For $N$ acting on $(\mathcal{T} \cdot \delta)$, consider the complex $\left(\right.$ Cone $\left.(N)^{\cdot}, D\right)$ with differential $D(\alpha, \beta)=(\delta(\alpha), N(\beta)-\delta(\beta))$.

(1) For $2 r+m>0$, the map $N^{-(2 r+m)}$ gives an isomorphism between the cohomology group in $\mathbb{H}^{\cdot}\left(\operatorname{Ker}(N)^{\circ}\right)$, which lies over the point of coordinates $(2 r, 2 r+m)$ in Figure 1 and the cohomology group in $\mathbb{H} \cdot\left(\operatorname{Coker}(N)^{\cdot}\right)$ that lies over the point of coordinates $(-2(r+m), 0)$ in Figure 1 .

(2) In the range $2 r+m<-1$, the cohomology $\mathbb{H} \cdot\left(\right.$ Cone $\left.(N)^{\prime}, D\right)$ is identified with $\mathbb{H}^{+}+1(\operatorname{Coker}(N), \delta)$.

(3) Upon identifying the variable $U^{-1}$ with the Tate twist by $2 \pi \sqrt{-1}$, and for a for fixed $r \in \mathbb{Z}_{\leq 0}$, we obtain quasi isomorphic complexes

$$
\left.\left(\text { Cone }(N)^{\cdot}, D\right)\right|_{U^{r}=(2 \pi \sqrt{-1})^{-r}} \simeq\left(C_{\mathcal{D}}^{*}(-r), d_{\mathcal{D}}\right)
$$

3.2. Local factors. The "archimedean factor" (i.e. the local factor at arithmetic infinity) $L_{\kappa}\left(H^{m}, s\right)$ is a product of powers of shifted Gamma functions, with exponents and arguments that depend on the Hodge structure on $H^{m}=H^{m}(X, \mathbb{C})=\oplus_{p+q=m} H^{p, q}$. More precisely, it is given by (cf. [25]

$$
L_{\kappa}\left(H^{m}, s\right)= \begin{cases}\prod_{p, q} \Gamma_{\mathbb{C}}(s-\min (p, q))^{h^{p, q}} & \kappa=\mathbb{C} \\ \prod_{p<q} \Gamma_{\mathbb{C}}(s-p)^{h^{p, q}} \prod_{p} \Gamma_{\mathbb{R}}(s-p)^{h^{p+}} \Gamma_{\mathbb{R}}(s-p+1)^{h^{p-}} & \kappa=\mathbb{R},\end{cases}
$$

where the $h^{p, q}$, with $p+q=m$, are the Hodge numbers, $h^{p, \pm}$ is the dimension of the $\pm(-1)^{p}$-eigenspace of de Rham conjugation on $H^{p, p}$, and

$$
\Gamma_{\mathbb{C}}(s):=(2 \pi)^{-s} \Gamma(s) \quad \Gamma_{\mathbb{R}}(s):=2^{-1 / 2} \pi^{-s / 2} \Gamma(s / 2) .
$$

It is shown in [1] that the local factor (3.14) can be computed as a Ray-Singer determinant

$$
L_{\kappa}\left(H^{m}, s\right)=\operatorname{det}_{\infty}\left(\left.\frac{1}{2 \pi}(s-\Phi)\right|_{H_{a r}^{m}(X)}\right)^{-1}
$$


where the "archimedean cohomology" $H_{a r}^{m}(X)$ is an infinite-dimensional real vector space, and the zeta regularized determinant of an unbounded self adjoint operator $T$ is defined as

$$
\operatorname{det}_{\infty}(s-T)=\exp \left(-\left.\frac{d}{d z} \zeta_{T}(s, z)\right|_{z=0}\right)
$$

In [9] (cf. §5) the archimedean cohomology is identified with the "inertia invariants"

$$
H_{a r}^{\cdot}(X)=\mathbb{H}^{\cdot}\left(K^{\cdot}, d^{\prime}+d^{\prime \prime}\right)^{N=0},
$$

where $\mathbb{H}^{\cdot}\left(K^{\prime}, d^{\prime}+d^{\prime \prime}\right)$ is the hypercohomology of the complex (3.1) and $\mathbb{H}^{\cdot}\left(K^{\prime}, d^{\prime}+d^{\prime \prime}\right)^{N=0}$ is the kernel of the map induced in hypercohomology by the monodromy $N$. This follows the expectation that the fiber over arithmetic infinity has semi-stable reduction.

At arithmetic infinity, the alternating product of the local factors of $X$ can be described in terms of the operators $\sigma^{L}(w)^{2}$ and $\Phi\left(c f\right.$. [10] par. 3.4). Let $\Phi_{0}$ denote the restriction of the operator $\Phi$ to $\mathbb{H}^{\cdot}\left(K^{\cdot}\right)^{N=0}$. For $a$ a bounded operator on $\mathbb{H}^{\cdot}\left(K^{\cdot}\right)^{N=0}$, let $\zeta_{a, \Phi_{0}}(s, z)$ denote the two variable zeta function

$$
\zeta_{a, \Phi_{0}}(s, z)=\sum_{\lambda \in \operatorname{Spec}\left(\Phi_{0}\right)} \operatorname{Tr}\left(a \Pi_{\lambda}\right)(s-\lambda)^{-z}
$$

where $\Pi_{\lambda}$ are the spectral projections of $\Phi_{0}$. Let $\operatorname{det}_{\infty, a, \Phi_{0}}$ denote the zeta regularized determinant

$$
\operatorname{det}_{\infty, a, \Phi_{0}}(s)=\exp \left(-\left.\frac{d}{d z} \zeta_{a, \Phi_{0}}(s, z)\right|_{z=0}\right) .
$$

Proposition 3.3. The two variable zeta function $\zeta_{\sigma^{L}(w)^{2}, \Phi_{0}}(s, z)$ satisfies

$$
\operatorname{det}_{\infty, \sigma^{L}(w)^{2}, \Phi_{0} /(2 \pi)}\left(\frac{s}{2 \pi}\right)^{-1}=\prod_{m=0}^{2 n} L_{\mathbb{C}}\left(H^{m}, s\right)^{(-1)^{m+n}} .
$$

Proof. The operator $\Phi_{0}$ has spectrum

$$
\operatorname{Spec}\left(\Phi_{0}\right)=\left\{\lambda_{\ell, p, q}=\min \{p, q\}-\ell: \ell \in \mathbb{Z}_{\geq 0}\right\},
$$

with eigenspaces $E_{\ell, p, q}=H^{p, q}(X) \otimes U^{r} \otimes \hbar^{2 r+m}$, with $r=\ell-\min \{p, q\}$ and $m=p+q$. In fact, for $\mathbb{H}^{\prime}(K)^{N=0}$ we have $2 r+m=k$, and $k \geq|p-q|$ so that $r \geq-\min \{p, q\}$. The result then follows as in [10] $\S 3.3$.

\section{Archimedean COHOMOlogy AND NEARby CyCles}

The definition of the complex (3.1) was inspired by an analogy with the resolution of the complex of nearby cycles associated to an analytic degeneration with normal crossings over a disk, cf. [28]. In this section we recall this classical construction and we relate it to its archimedean counterpart by making the analogy more explicit.

4.1. The complex of nearby cycles. Let $\mathfrak{X}$ and $\Delta$ be complex analytic manifolds, of complex dimensions $\operatorname{dim} \mathfrak{X}=n+1$ and $\operatorname{dim} \Delta=1$, and let $f: \mathfrak{X} \rightarrow \Delta$ be a flat, proper morphism with projective fibers. For $0 \in \Delta$, we write $Y=f^{-1}(0), \mathfrak{X}^{*}=\mathfrak{X} \backslash Y$, and $\Delta^{*}=\Delta \backslash\{0\}$. We assume that the map $f$ is smooth on $\mathfrak{X}^{*}$ and that $Y$ is a divisor with normal crossings on $\mathfrak{X}$. Under these hypotheses, the relative de Rham complex of sheaves of differential forms with logarithmic poles along $Y$ is well defined and of the form

$$
\Omega_{\mathfrak{X} / \Delta}^{m}(\log Y):=\wedge^{m} \Omega_{\mathfrak{X} / \Delta}^{1}(\log Y),
$$

where we use the inclusion $f^{*} \Omega_{\Delta}^{1}(\log 0) \subset \Omega_{\mathfrak{X}}^{1}(\log Y)$ to define

$$
\Omega_{\mathfrak{X} / \Delta}^{1}(\log Y):=\Omega_{\mathfrak{X}}^{1}(\log Y) / f^{*} \Omega_{\Delta}^{1}(\log 0) .
$$


Consider local coordinates $\left\{z_{0}, \ldots z_{n}\right\}$ at $P \in Y$, with $t$ the local coordinate at $0 \in \Delta$, so that $t \circ f=z_{0}^{e_{0}} \cdots z_{k}^{e_{k}}$ for some $0 \leq k \leq n$ and $e_{i} \in \mathbb{N}$. The stalk $\Omega_{\mathfrak{X} / \Delta}^{1}(\log Y)_{P}$ is the $\mathcal{O}_{\mathfrak{X}, P}$-module with generators $\left\{d z_{0} / z_{0}, \ldots, d z_{k} / z_{k}, d z_{k+1}, \ldots d z_{n}\right\}$ satisfying the relation $\sum_{i=0}^{k} e_{i} d z_{i} / z_{i}=0$. Thus, $\Omega_{\mathfrak{X} / \Delta}^{1}(\log Y)$ is a locally free sheaf of rank $n=\operatorname{dim} \mathfrak{X}-1$ endowed with differential $d$ given by the composite

$$
d: \mathcal{O}_{\mathfrak{X}} \rightarrow \Omega_{\mathfrak{X}}^{1} \hookrightarrow \Omega_{\mathfrak{X}}^{1}(\log Y) \rightarrow \Omega_{\mathfrak{X} / \Delta}^{1}(\log Y),
$$

where $\Omega_{\mathfrak{X}}^{1}(\log Y)$ is the free sheaf of $\mathcal{O}_{\mathfrak{X}}$-modules on the same generators of $\Omega_{\mathfrak{X} / \Delta}^{1}(\log Y)$.

The relative hypercohomology sheaves $\mathbb{R}^{m} f_{*} \Omega_{\mathfrak{X} / \Delta}(\log Y)$ are locally free $\mathcal{O}_{\Delta}$-modules of finite rank (cf. [28). This follows from the fact that the restriction $f: \mathfrak{X}^{*} \rightarrow \Delta^{*}$, which is a smooth fiber bundle, determines, for all $s \in \Delta^{*}$, a canonical isomorphism of complexes

$$
\Omega_{\mathfrak{X} / \Delta}(\log Y) \otimes_{\mathcal{O}_{\mathfrak{X}}} \mathcal{O}_{\mathfrak{X}_{s}} \stackrel{\simeq}{\rightarrow} \Omega_{\mathfrak{X}_{s}}
$$

on $\mathfrak{X}_{s}=f^{-1}(s)$. This implies that $\Omega_{\mathfrak{X} / \Delta}(\log Y) \otimes_{\mathcal{O}_{\mathfrak{X}}} \mathcal{O}_{\mathfrak{X}_{s}}$ is a resolution of the constant sheaf $\mathbb{C}$ on $\mathfrak{X}_{s}$, hence

$$
\mathbb{H}^{m}\left(\mathfrak{X}_{s}, \Omega_{\mathfrak{X} / \Delta}(\log Y) \otimes_{\mathcal{O}_{\mathfrak{X}}} \mathcal{O}_{\mathfrak{X}_{s}}\right) \simeq H^{m}\left(\mathfrak{X}_{s}, \mathbb{C}\right), \quad \forall s \in \Delta^{*},
$$

hence the complex dimension of $\mathbb{H}^{m}\left(\mathfrak{X}_{s}, \Omega_{\mathfrak{X} / \Delta}(\log Y) \otimes_{\mathcal{O}_{\mathfrak{X}}} \mathcal{O}_{\mathfrak{X}_{s}}\right)$ is a locally constant function. One obtains

$$
\mathbb{R}^{m} f_{*} \Omega_{\mathfrak{X} / \Delta}(\log Y) \otimes_{\mathcal{O}_{\Delta^{*}}} k(s) \simeq H^{m}\left(\mathfrak{X}_{s}, \mathbb{C}\right), \quad \forall s \in \Delta^{*},
$$

so that $\mathbb{R}^{m} f_{*} \Omega_{\mathfrak{X} / \Delta}(\log Y)$ is a locally free $\mathcal{O}_{\Delta^{*}}$-module of finite rank. Moreover, if $\Delta$ is a small disk, there exists an isomorphism

$$
H^{m}\left(\tilde{\mathfrak{X}}^{*}, \mathbb{C}\right) \stackrel{\simeq}{\rightarrow} \mathbb{H}^{m}\left(Y, \Omega_{\mathfrak{X} / \Delta}(\log Y) \otimes_{\mathcal{O}_{\mathfrak{X}}} \mathcal{O}_{Y}\right)
$$

where $\tilde{\mathfrak{X}}^{*}=\mathfrak{X} \times_{\Delta} \tilde{\Delta}^{*}$ and $\tilde{\Delta}^{*} \rightarrow \Delta^{*}$ is the universal covering space of $\Delta^{*}=\Delta \backslash\{0\}$, so that $\operatorname{dim} \mathbb{H}^{m}\left(\mathfrak{X}_{s}, \Omega_{\mathfrak{X} / \Delta}(\log Y) \otimes_{\mathcal{O}_{\mathfrak{X}}} \mathcal{O}_{\mathfrak{X}_{s}}\right)$ is locally constant on $\Delta$.

A stronger result $(c f .[28, \S 9)$ shows that the Gauss-Manin connection

$$
\nabla: \mathbb{R}^{m} f_{*} \Omega_{\mathfrak{X} / \Delta}(\log Y) \rightarrow \Omega_{\Delta}^{1}(\log 0) \otimes_{\mathcal{O}_{\Delta}} \mathbb{R}^{m} f_{*} \Omega_{\mathfrak{X} / \Delta}(\log Y)
$$

has logarithmic singularities at $0 \in \Delta$ and in case of an algebraic morphism $f$ admits an algebraic description. In particular the residue of $\nabla$ at zero is a well defined operator

$$
N:=\operatorname{Res}_{0}(\nabla)
$$

in fact it is an endomorphism of $H^{m}\left(\tilde{\mathfrak{X}}^{*}, \mathbb{C}\right)$. The eigenvalues of $\operatorname{Res}_{0}(\nabla)$ are rational numbers $\alpha$ with $0 \leq \alpha<1$. The monodromy transformation $T$ induces an automorphism $T_{0}$ of (4.1) and it can be shown that

$$
T_{0}=\exp \left(-2 \pi \sqrt{-1} \operatorname{Res}_{0}(\nabla)\right) .
$$

It follows that the eigenvalues of $T_{0}$ are roots of unity, so that a power $T_{0}^{d}$ is unipotent. In fact, up to a base change $\Delta \rightarrow \Delta, z \mapsto z^{d}$, one can assume that $T_{0}$ is already unipotent, this means that the residue (4.4) is nilpotent.

There are two important filtrations on the cohomology $H^{m}\left(\tilde{\mathfrak{X}}^{*}, \mathbb{C}\right)$. One is the Hodge filtration $F^{p} H^{m}\left(\tilde{\mathfrak{X}}^{*}, \mathbb{C}\right)$ determined by the isomorphism (4.2) and the 'naive filtration' on $\Omega_{\mathfrak{X} / \Delta}(\log Y) \otimes_{\mathcal{O}_{\mathfrak{X}}} \mathcal{O}_{Y}$.

The other is the Picard-Lefschetz filtration $L_{\ell} H^{m}\left(\tilde{\mathfrak{X}}^{*}, \mathbb{C}\right), \ell \in \mathbb{Z}$, which is a canonical, finite, increasing filtration associated to the map $N$ and defined by induction. The properties and behavior of this filtration are a priori rather mysterious as its definition is given via an "indirect method". The main result in 28 shows that the data $\left(H^{*}\left(\tilde{\mathfrak{X}}^{*}, \mathbb{C}\right), L ., F^{*}\right)$ determine a mixed $\mathbb{Q}$-Hodge structure on $H \cdot\left(\tilde{\mathfrak{X}}^{*}, \mathbb{Q}\right)$.

This result is obtained by studying "explicitly" the Picard-Lefschetz filtration $L$. on a resolution of the complex of sheaves $\Omega_{\mathfrak{X} / \Delta}(\log Y) \otimes_{\mathcal{O}_{\mathfrak{X}}} \mathcal{O}_{Y^{\text {red }}}$ of the form

$$
A^{s, k}:=\Omega_{\mathfrak{X}}^{s+k+1}(\log Y) / W_{k} \Omega_{\mathfrak{X}}^{s+k+1}(\log Y), \quad s, k \in \mathbb{Z}_{\geq 0},
$$


where the weight filtration $W$. on $\Omega_{\mathfrak{X}}(\log Y)$ is defined as

$$
W_{k} \Omega_{\mathfrak{X}}^{m}(\log Y):=\Omega_{\mathfrak{X}}^{k}(\log Y) \wedge \Omega_{\mathfrak{X}}^{m-k},
$$

and the differentials

$$
d^{\prime}: A^{s, k} \rightarrow A^{s+1, k}, \quad d^{\prime \prime}: A^{s, k} \rightarrow A^{s, k+1} .
$$

on (4.6) are the usual differential $d^{\prime}$ on $\Omega_{\mathfrak{X}}(\log Y)$ and

$$
d^{\prime \prime}(\alpha)=(-1)^{s} \alpha \wedge \theta, \quad \text { for } \quad \theta=f^{*}\left(\frac{d t}{t}\right) .
$$

Notice that $\theta$ can be seen as an element of

$$
H^{1}\left(\mathfrak{X}^{*}, \mathbb{Q}\right)(1)=2 \pi \sqrt{-1} H^{1}\left(\mathfrak{X}^{*}, \mathbb{Q}\right),
$$

because the form $d t / t$ on $\Delta^{*}$ has period $2 \pi \sqrt{-1}$, so that

$$
(2 \pi \sqrt{-1})^{-1} d t / t \in H^{1}\left(\Delta^{*}, \mathbb{Z}\right) \subset H^{1}\left(\Delta^{*}, \mathbb{Q}\right) .
$$

Wedging with $\theta$ provides an injective map

$$
\wedge \theta: \Omega_{\mathfrak{X} / \Delta}^{m}(\log Y) \hookrightarrow \Omega_{\mathfrak{X}}^{m+1}(\log Y)
$$

and an induced morphism of complexes of sheaves $\phi: \Omega_{\mathfrak{X} / \Delta}(\log Y) \otimes_{\mathcal{O}_{\mathfrak{X}}} \mathcal{O}_{Y^{\text {red }}} \rightarrow A$, which defines a resolution of the unipotent factor of the complex of nearby cycles, with $\left(A, \delta=d^{\prime}+d^{\prime \prime}\right)$ the total complex of (4.6). The endomorphism $\nu^{s, k}: A^{s, k} \rightarrow A^{s-1, k+1}$ given by the natural projection on forms is non-trivial because of the presence of the cutoff by the weight filtration $W_{k}$ on $\Omega_{\mathfrak{X}}^{m}(\log Y)$ and it plays a central role in this theory as it describes the local monodromy map on the resolution $A$.

It can be shown (see [18]) that the map induced in hypercohomology by

$$
\tilde{\nu}=(-1)^{s} \nu^{s, k}: A^{s, k} \rightarrow A^{s-1, k+1},
$$

satisfying $\tilde{\nu} \delta+\delta \tilde{\nu}=0$, is the residue (4.4) of the Gauss-Manin connection,

$$
N: H^{m}\left(\tilde{\mathfrak{X}}^{*}, \mathbb{C}\right) \rightarrow H^{m}\left(\tilde{\mathfrak{X}}^{*}, \mathbb{C}\right) .
$$

More precisely, $N$ is obtained as the connecting homomorphism in the long exact sequence of hypercohomology associated to the exact sequence of complexes of sheaves on $Y$

$$
0 \rightarrow A \cdot[-1] \stackrel{\epsilon}{\rightarrow} \text { Cone }(\tilde{\nu}) \stackrel{\eta}{\rightarrow} A \cdot \rightarrow 0 .
$$

The complex $\left(\operatorname{Cone}^{\cdot}(\tilde{\nu}), D\right), D=(\delta+\tilde{\nu}, \delta)$ is quasi-isomorphic to $\Omega_{\mathfrak{X}}(\log Y) \otimes_{\mathcal{O}_{\mathfrak{X}}} \mathcal{O}_{Y^{\text {red }}}$. The map $\tilde{\nu}$ measures the difference between differentiation in Cone $(\tilde{\nu})$ and in $A$ which appears when one considers the section of $\eta: A^{s, k} \rightarrow \operatorname{Cone}^{s, k}(\tilde{\nu})=A^{s-1, k} \oplus A^{s, k}$.

4.2. Nearby cycles at arithmetic infinity. The main idea that motivates the definition of the complex (3.1) is to "transfer" these results to the archimedean setting, where one deals with a smooth, projective algebraic variety $X$ over $\mathbb{C}$ or $\mathbb{R}$, interpreted as the generic fiber of a degeneration "around" infinity. The main aspects of the above construction that one wishes to retain are the fact that the cutoff by $W_{k}$ on $\Omega_{\mathfrak{X}}^{m}(\log Y)$ is introduced because the complex of the nearby cycles is the restriction to $Y$ of the complex $\Omega_{\mathfrak{X} / \Delta}^{m}(\log Y)$. It is the presence of this cutoff that makes the morphism $\nu^{s, k}$ non-trivial on $A^{s, k}$. Moreover, another essential observation is that translates of the weight filtration

$$
L_{\ell} A^{s, k}=W_{2 k+\ell+1} \Omega_{\mathfrak{X}}^{s+k+1}(\log Y) / W_{k} \Omega_{\mathfrak{X}}^{s+k+1}(\log Y) ; \quad \ell \in \mathbb{Z}
$$

and the corresponding graded spaces

$$
g r_{\ell}^{L} A^{s, k}= \begin{cases}g r_{2 k+\ell+1}^{W} \Omega_{\mathfrak{X}}^{s+k+1}(\log Y) & \ell+k \geq 0 \\ 0 & \ell+k<0\end{cases}
$$

describe the strata of the special fiber $Y$, through the Poincaré residue map

$$
\text { Res : } W_{k} \Omega_{\mathfrak{X}}^{m}(\log Y) \rightarrow\left(a_{k}\right)_{*} \Omega_{\tilde{Y}^{(k)}}^{m-k},
$$


where

$$
\tilde{Y}^{(k)}:=\coprod_{1 \leq i_{1}<\ldots<i_{k} \leq M} Y_{i_{1}} \cap \ldots \cap Y_{i_{k}}
$$

is the $k$-th stratum of $Y=Y_{1} \cup \ldots \cup Y_{M}$ and $\left(a_{k}\right)_{*}: \tilde{Y}^{(k)} \rightarrow \mathfrak{X}$ is the canonical projection. The Poincaré residue (4.14) is given by

$$
\operatorname{Res}\left(\sum_{1 \leq i_{1}<\ldots<i_{k} \leq K} \omega_{i_{1} \ldots i_{k}} \frac{d z_{i_{1}}}{z_{i_{1}}} \wedge \ldots \wedge \frac{d z_{i_{k}}}{z_{i_{k}}}\right)=\sum_{1 \leq i_{1}<\ldots<i_{k} \leq K} \operatorname{res}\left(\omega_{i_{1} \ldots i_{k}}\right),
$$

where $z_{1} \cdots z_{K}=0$ is the local description of $Y^{\text {red }}$ (the closed subset $Y$ of $\mathfrak{X}$ with its reduced scheme structure), $\omega_{i_{1} \ldots i_{k}}$ is a section of $\Omega_{\mathfrak{X}}^{m-k}$, and res : $\Omega_{\mathfrak{X}}^{m-k} \rightarrow\left(a_{k}\right)_{*} \Omega_{\tilde{Y}(k)}^{m-k}$ is the restriction to the stratum $\tilde{Y}^{(k)}$.

This means that it is sufficient to provide a version at arithmetic infinity of the weight filtration $W$. and the Picard-Lefschetz filtration $L$., as these are sufficient to characterize the geometry of the singular fiber $Y=f^{-1}(0)$, which is strictly related to the behavior of the monodromy map.

Notice that the period $2 \pi \sqrt{-1}$ of the form $d t / t$ on $\Delta^{*}$ (cf. (4.8), (4.9), (4.10) ) corresponds to a Tate twist on the (rational) cohomology of the generic fiber $\tilde{\mathfrak{X}}^{*}$. It is important to stress the fact that this 'detects' the presence of the singular fiber through an operation that does not involve $Y$ explicitly, and therefore can be transported at arithmetic infinity (where the description of the fiber 'over infinity' is still mysterious) on a complex of real differential forms. Moreover, the fact that the cutoff by $W_{k}$ on $\Omega_{x}^{m}(\log Y)$ implies the non-triviality of the monodromy map $\nu^{s, k}$ suggests a definition of the monodromy operator $N$ "at infinity" in terms of an analogous weight filtration on a complex of Tate-twisted real differential forms.

By weight of a real $m$-form

$$
\alpha \in \bigoplus_{p+q=m}\left(\Omega_{X}^{p, q}+\Omega_{X}^{q, p}\right) \mathbb{R}
$$

on $X$ we mean the non-negative integer $|p-q|$. At arithmetic infinity, the operations of taking the residues and hence considering holomorphic differential forms on the strata of $Y$ can be rephrased in a form suitable to be included in the definition of the complex $K^{*}$ of (3.1).

In fact, one should interpret the archimedean complex (3.1) as a "filtered copy" of $A^{s, k}$, with an additional condition characterizing the graded pieces $g r{ }^{L} A^{*}$. In fact, in the case of the complex of the nearby cycles, there is a graded isomorphism

$$
g r_{\ell}^{L} A \simeq \bigoplus_{k \geq \max (0,-\ell)}\left(a_{2 k+\ell+1}\right)_{*} \Omega_{\tilde{Y}(2 k+\ell+1)}^{+1}[-\ell-2 k-1]
$$

under the condition $\ell+k \geq 0$. Furthermore, in $g r_{\ell}^{L} A$ the second differential is trivial, $d^{\prime \prime}=0$. The induced weights spectral sequence

$$
E_{1}^{i, m-i}=\bigoplus_{k \geq \max (0, i)} H^{m+i-2 k}\left(\tilde{Y}^{(2 k-i+1)}, \mathbb{Q}\right)(i-k) \Rightarrow H^{m}\left(\tilde{\mathfrak{X}}^{*}, \mathbb{Q}\right)
$$

degenerates at the $E_{2}$ term. The $E_{1}^{i, m-i}$ term is a pure Hodge structure of weight $m-i$. A major result in the theory shows that the filtration induced on the abutment coincides with the Picard-Lefschetz filtration.

At arithmetic infinity, in terms of the complex (3.1), for

$$
K^{i, j}=\bigoplus_{k \geq \max \{0, i\}} K^{i, j, k}
$$


the terms

$$
K^{i, m-n, k}= \begin{cases}\bigoplus_{\substack{p+q=m \\|p-q| \leq 2 k-i}}\left(\Omega_{X}^{p, q} \oplus \Omega_{X}^{q, p}\right)_{\mathbb{R}}\left(\frac{m-i}{2}\right) & \text { if } m-i \equiv 0(2), k \geq \max (0, i) \\ 0 & \text { otherwise }\end{cases}
$$

give the archimedean analog, at the level of the real differential forms, of the $E_{1}^{i, m-i}$-term of the spectral sequence (4.17). At arithmetic infinity the weight is $i-m$.

These analogies suggest a geometric interpretation of the indices involved in the definition of (3.1). The first index is associated to the $\ell$-th piece of an "archimedean monodromy filtration" $L_{\ell}$ on $\oplus_{p+q=m}\left(\Omega_{X}^{p, q} \oplus \Omega_{X}^{q, p}\right)_{\mathbb{R}}$, with $\ell=-i$. This explains our previous comment that the archimedean complex should be thought of as a filtered copy of $A^{s, k}$. The cutoffs $|p-q| \leq 2 k-i$ and $k \geq \max \{0, i\}$ correspond to considering the filtered piece $L_{\ell} A^{s, k}=W_{2 k+\ell+1} A^{s, k}$ in Steenbrink's theory with the cutoff by $W_{k}$ on $\Omega_{X}^{s+k+1}(\log Y)$, justified by restricting (relative) differential forms to the special fiber. In this identification the third index $k$ of (3.1) plays the role of the index $k$ of the anti-holomorphic forms in the double complex (4.6). The archimedean theory is a weighted "even theory" since $i-m=2 r$. The second index $j=m-n$ detects the total degree $m$ of the differential forms.

Finally, we remark that there is a fundamental difference in the definition of the differentials (3.2) (3.3) in the complex (3.1) at infinity and their geometric analogs (4.7). In fact, while the differential $d^{\prime}$ is similar in both theories, in the geometric case, the action of $d^{\prime \prime}$ by the wedging with the form $\theta=f^{*}(d t / t)$ involves a Tate twist on the rational version of the complex $A^{s, k}$, the differential $d^{\prime \prime}$ in the archimedean case does not involve any twist.

\section{Monodromy AND the RenORmalization Group}

In the construction at arithmetic infinity we obtain an analog of the formula (4.4) for the logarithm of the monodromy $N$ as the residue of a connection, in terms of a Birkhoff decomposition of loops and a Riemann-Hilbert problem analogous to those underlying the theory of renormalization in QFT, as developed by Connes and Kreimer ([7] [5], $c f$. also [ [] $)$.

In our case, the Birkhoff decomposition will take place in the group $G$ of automorphisms of the complex $\left(\mathcal{T}_{\mathbb{C}}, \delta\right)$, where $\mathcal{T}_{\mathbb{C}}$ is the complexification of $\mathcal{T}$. The Birkhoff decomposition will determine a one parameter family of principal $G$-bundles $\mathcal{P}_{\mu}$ on $\mathbb{P}^{1}(\mathbb{C})$, with trivializations

$$
\phi_{\mu}(z)=\phi_{\mu}^{-}(z)^{-1} \phi_{\mu}^{+}(z), \quad z \in \partial \Delta \subset \mathbb{P}^{1}(\mathbb{C}), \quad \mu \in \mathbb{C}^{*},
$$

where $\phi_{\mu}^{+}$is a holomorphic function on a disk $\Delta$ around $z=0$ and $\phi_{\mu}^{-}$is holomorphic on $\mathbb{P}^{1}(\mathbb{C}) \backslash \Delta$, normalized by $\phi_{\mu}^{-}(\infty)=1$. The parameter $\mu$ is related to a scaling action by $\mathbb{R}_{+}^{*}$, by $\mu \mapsto \lambda \mu$. We shall construct the data (5.1) in such a way that the negative part of the Birkhoff decomposition $\phi_{\mu}^{-}=\phi^{-}$is in fact independent of $\mu$, as in the theory of renormalization. As part of the data, one also considers a one parameter group of automorphisms $\theta$, and the corresponding infinitesimal generator $\Upsilon=\left.\frac{d}{d t} \theta_{t}\right|_{t=0}$, so that

$$
\phi_{\lambda \mu}(\epsilon)=\theta_{t \epsilon} \phi_{\mu}(\epsilon), \quad \forall \lambda=e^{t} \in \mathbb{R}_{+}^{*}, \quad \epsilon \in \partial \Delta .
$$

The corresponding renormalization group is the one parameter semigroup

$$
\rho(\lambda)=\lim _{\epsilon \rightarrow 0} \phi^{-}(\epsilon) \theta_{t \epsilon}\left(\phi^{-}(\epsilon)^{-1}\right), \quad \forall \lambda=e^{t} \in \mathbb{R}_{+}^{*} .
$$

Following [7, one can write $\phi^{-}(z)$ in the form

$$
\phi^{-}(z)^{-1}=1+\sum_{k=1}^{\infty} \frac{d_{k}}{z^{k}},
$$

with coefficients

$$
d_{k}=\int_{s_{1} \geq \cdots \geq s_{k} \geq 0} \theta_{-s_{1}}(\beta) \cdots \theta_{-s_{k}}(\beta) d s_{1} \cdots d s_{k} .
$$


Here $\beta$ is the beta-function of renormalization, related to the residue at zero of $\phi$ by

$$
\beta=\Upsilon \operatorname{Res} \phi,
$$

where $\Upsilon$ is the generator of $\theta_{t}$ and the residue is defined as

$$
\operatorname{Res} \phi=\left.\frac{d}{d z}\left(\phi^{-}(1 / z)^{-1}\right)\right|_{z=0}
$$

In the construction at arithmetic infinity, the grading operator $\Phi$ induces a time evolution on the complex $\left(\mathcal{T}_{\mathbb{C}}, \delta\right)$ given by the "Frobenius flow" (2.28),

$$
F_{t}=e^{t \Phi}, \quad t \in \mathbb{R},
$$

and we denote by $\theta_{t}$ the induced time evolution on $\operatorname{End}\left(\mathcal{T}_{\mathbb{C}}, \delta\right)$,

$$
\theta_{t}(a)=e^{-t \Phi} a e^{t \Phi}
$$

with the corresponding $\Upsilon$ given by

$$
\Upsilon(a)=\left.\frac{d}{d t} \theta_{t}(a)\right|_{t=0}=[a, \Phi]
$$

The analogy with the complex of nearby cycles of a geometric degeneration over a disk suggests to make the following prescription for the residue of $\phi$ :

$$
\operatorname{Res} \phi=N \text {. }
$$

As in the case of Connes-Kreimer, the residue uniquely determines $\phi^{-}$via (5.5) and (5.4). We obtain the following result.

Theorem 5.1. There is a unique holomorphic map $\phi^{-}: \mathbb{P}^{1}(\mathbb{C}) \backslash\{0\} \rightarrow$ Aut $\left(\mathcal{T}_{\mathbb{C}}\right)$ satisfying (5.4), with coefficients (5.5) and residue (5.11), and it is of the form

$$
\phi^{-}(z)=\exp (-N / z) \text {. }
$$

Proof. First notice that the time evolution (5.9) satisfies

$$
\theta_{t}(N)=e^{t} N
$$

Moreover, by (5.10) and (5.11), we have

$$
\beta=[N, \Phi]=N
$$

Thus, we can write the coefficients $d_{k}$ of (5.5) in the form

$$
d_{k}=N^{k} \int_{s_{1} \geq \cdots \geq s_{k} \geq 0} e^{-\left(s_{1}+\cdots+s_{k}\right)} d s_{1} \cdots d s_{k} .
$$

It is easy to see by induction that

$$
u_{k}(t):=\int_{0}^{t} \int_{0}^{s_{1}} \cdots \int_{0}^{s_{k-1}} e^{-\left(s_{1}+\cdots+s_{k}\right)} d s_{1} \cdots d s_{k}=\frac{\left(1-e^{-t}\right)^{k}}{k !},
$$

satisfying the recursion $u_{k+1}^{\prime}(t)=e^{-t} u_{k}(t)$, so that

$$
\int_{s_{1} \geq \cdots \geq s_{k} \geq 0} e^{-\left(s_{1}+\cdots+s_{k}\right)} d s_{1} \cdots d s_{k}=\frac{1}{k !} .
$$

This implies that $d_{k}=N^{k} / k$ ! , hence we obtain that the series (5.4) is just

$$
\phi^{-}(z)^{-1}=\sum_{k=0}^{\infty} \frac{z^{-k}}{k !} N^{k}
$$

and $\phi^{-}(z)=\exp (-N / z)$. 
Correspondingly, we see that the renormalization group (5.3) is given by

$$
\rho(\lambda)=\lambda^{N}=\exp (t N), \quad \forall \lambda=e^{t} \in \mathbb{R}_{+}^{*},
$$

since we have

$$
\theta_{t \epsilon}\left(\phi^{-}(\epsilon)\right)=\exp \left(-\frac{\lambda^{\epsilon}}{\epsilon} N\right) \quad \forall \lambda=e^{t} \in \mathbb{R}_{+}^{*} .
$$

We now show that the other term of the Birkhoff decomposition (5.1) is determined by the requirement (5.2) of compatibility between the scaling and the time evolution.

Theorem 5.2. Consider the holomorphic map $\phi_{\mu}^{+}: \mathbb{P}^{1}(\mathbb{C}) \backslash\{\infty\} \rightarrow \operatorname{Aut}\left(\mathcal{T}_{\mathbb{C}}\right)$ given by

$$
\phi_{\mu}^{+}(z)=\exp \left(\frac{\mu^{z}-1}{z} N\right) .
$$

The loop $\phi_{\mu}(z)=\phi^{-}(z)^{-1} \phi_{\mu}^{+}(z)$ with $\phi^{-}(z)=\exp (-N / z)$ and $\phi_{\mu}^{+}$as in (5.17) satisfies the relation (5.2).

Proof. First notice that (5.17) is indeed holomorphic at $z=0$ with $\phi_{\mu}^{+}(0)=\exp (\mu N)$. By (5.1) and (5.16), the relation (5.2) is equivalent to requiring that, for all $\lambda=e^{t} \in \mathbb{R}_{+}^{*}$, the function $\phi_{\mu}^{+}$satisfies

$$
\phi_{\lambda \mu}^{+}(\epsilon)=\exp \left(\frac{\lambda^{\epsilon}-1}{\epsilon} N\right) \theta_{t \epsilon}\left(\phi_{\mu}^{+}(\epsilon)\right) \text {. }
$$

For $\phi_{\mu}^{+}$as in (5.17) we have

$$
\theta_{t \epsilon}\left(\phi_{\mu}^{+}(\epsilon)\right)=\exp \left(\frac{\mu^{\epsilon}-1}{\epsilon} \lambda^{\epsilon} N\right)=\exp \left(\frac{(\lambda \mu)^{\epsilon}-1}{\epsilon} N\right) \exp \left(\frac{1-\lambda^{\epsilon}}{\epsilon} N\right),
$$

so that (5.18) is satisfied.

Notice that, via the representation (2.24), it is possible to lift the Birkhoff decomposition (5.1) to a Birkhoff decomposition of the form

$$
g_{\mu}(z)=g^{-}(z)^{-1} g_{\mu}^{+}(z),
$$

where, with the notation of (2.22), we have

$$
g^{-}(z)=u(1 / z), \quad g_{\mu}(z)=u\left(\mu^{z} / z\right) .
$$

The renormalization group (5.15) then becomes simply the horocycle flow

$$
\rho(\lambda)=u(t)=\left(\begin{array}{cc}
1 & t \\
0 & 1
\end{array}\right) .
$$

There is a Riemann-Hilbert problem associated to the Birkhoff decomposition considered in the theory of renormalization ( $c f$. [8]). Namely, for $\gamma$ a generator of the fundamental group $\pi_{1}\left(\Delta^{*}\right)=\mathbb{Z}$ of the punctured disk, consider a complex linear representation $\pi: \mathbb{Z} \rightarrow G$. Under the assumption that the eigenvalues of $\pi(\gamma)$ satisfy

$$
0 \leq \operatorname{Re} \frac{\lambda}{2 \pi \sqrt{-1}}<1
$$

we can take the logarithm

$$
\frac{1}{2 \pi \sqrt{-1}} \log \pi(\gamma) .
$$

By the Riemann-Hilbert correspondence ( $c f$. 11), a representation $\pi: \mathbb{Z} \rightarrow G$ determines a bundle with connection $(\mathcal{E}, \nabla)$, where the Fuchsian connection $\nabla$ has local gauge potential on the disk $\Delta$ of the form

$$
-\phi^{+}(z)^{-1} \frac{\log \pi(\gamma) d z}{z} \phi^{+}(z)+\phi^{+}(z)^{-1} d \phi^{+}(z),
$$


with $\phi^{+}(z)$ the local trivialization of $\mathcal{E}$ over $\Delta$. The data $(\mathcal{E}, \nabla)$ correspond to a linear differential system $f^{\prime}(z)=A(z) f(z)$, with $\nabla f=d f-A(z) f d z$, for sections $f \in \Gamma\left(\Delta^{*}, \mathcal{E}\right)$.

In the case of the cohomological theory at arithmetic infinity, this amounts to a vector bundle $\mathcal{E}_{\mu}$ over $\mathbb{P}^{1}(\mathbb{C})$, with fiber $\mathcal{T}_{\mathbb{C}}$, associated to the principal $G$-bundles $\mathcal{P}_{\mu}$, with transition function the loop $\phi_{\mu}(z)$ and local trivializations given by the $\phi^{-}(z)$ and $\phi_{\mu}^{+}(z)$. We use the representation specified by

$$
\pi(\gamma):=\exp (-2 \pi \sqrt{-1} N),
$$

which is the analog of (4.5). This determines a one parameter family $\left(\mathcal{E}_{\mu}, \nabla_{\mu}\right)$ of linear differential systems over the disk $\Delta$, of the form

$$
\nabla_{\mu}: \mathcal{E}_{\mu} \rightarrow \mathcal{E}_{\mu} \otimes_{\mathcal{O}_{\Delta}} \Omega_{\Delta}(\log 0),
$$

where the connection on the restriction of $\mathcal{E}_{\mu}$ over $\Delta$ is given by

$$
\nabla_{\mu}=N\left(\frac{1}{z}+\frac{d}{d z} \frac{\mu^{z}-1}{z}\right) d z .
$$

Using the induced representation (2.24) in cohomology, this determines a corresponding linear differential system on the bundle of hypercohomologies $\mathbb{H}^{\cdot}\left(\mathcal{E}_{\mu}\right)$ with fiber $\mathbb{H}^{\cdot}\left(\mathcal{T}_{\dot{C}}\right)$,

$$
\nabla_{\mu}: \mathbb{H}^{\cdot}\left(\mathcal{E}_{\mu}^{\cdot}\right) \rightarrow \mathbb{H}^{\cdot}\left(\mathcal{E}_{\mu}\right) \otimes_{\mathcal{O}_{\Delta}} \Omega_{\Delta}(\log 0),
$$

which is the analog of the Gauss-Manin connection (4.3) in the geometric case. The connections (5.25) form an isomonodromic family, with

$$
\operatorname{Res}_{z=0} \nabla_{\mu}=N \text {. }
$$

\section{ReEs Sheaves at ARIThmetic INFINITy}

In the description (3.15) ( $c f$. 11]) of the archimedean factor of the Hasse-Weil L-function of the "motive" $H^{m}(X)$, for $X$ a smooth projective variety over a number field, the definition of the archimedean cohomology $H_{a r}^{m}(X)$ is motivated by previous work of J. M. Fontaine and it is expressed in terms of an additive functor $\mathbb{D}$ (derivation) from the (abelian) category of pure Hodge structures over $\kappa=\mathbb{C}, \mathbb{R}$ to the additive category whose objects are free modules of finite rank over the $\mathbb{R}$-algebra of polynomials in one variable endowed with a $\mathbb{R}$-linear endomorphism and satisfying certain properties. More precisely one sets

$$
H_{a r}^{m}(X)= \begin{cases}F i l^{0}\left(H_{B}^{m}(X, \mathbb{C}) \otimes_{\mathbb{C}} \mathbb{C}\left[z^{ \pm 1}\right]\right)^{c=i d} & \text { if } \kappa=\mathbb{C} \\ F i l^{0}\left(H_{B}^{m}(X, \mathbb{C}) \otimes_{\mathbb{C}} \mathbb{C}\left[z^{ \pm 1}\right]\right)^{c=i d, F_{\infty}=i d} & \text { if } \kappa=\mathbb{R}\end{cases}
$$

Here, $F i l^{q}$ denotes the filtration on the tensor product $H_{B}^{m}(X(\mathbb{C}), \mathbb{C}) \otimes_{\mathbb{C}} \mathbb{C}\left[z^{ \pm 1}\right]$ obtained from the Hodge filtration $F^{\cdot}$ on the Betti cohomology $H_{B}^{m}(X):=H_{B}^{m}(X(\mathbb{C}), \mathbb{C})$ and the one on the ring of Laurent polynomials $\mathbb{C}\left[z^{ \pm 1}\right]$ given by $F^{q} \mathbb{C}\left[z^{ \pm 1}\right]:=z^{-q} \mathbb{C}\left[z^{-1}\right], \forall q \in \mathbb{Z}$. By $c$ one denotes the conjugate linear involution (complex conjugation) and $F_{\infty}$ is a $\mathbb{C}$-linear involution (the infinite Frobenius).

The expectation is that one should obtain a description of the archimedean cohomology together with the linear "Frobenius flow" generated by $\Phi$ directly by some natural homological construction on a suitable non-linear dynamical system. In this direction, a more geometric construction of the archimedean cohomology was given in [13] ( $c f$. par. 3), where it was interpreted (for instance when $\kappa=\mathbb{C}$ ) as the space of global sections of a real analytic sheaf (Rees sheaf) $\zeta_{\mathbb{C}}^{\omega}\left(H^{m}(X), \gamma^{*}\right)$ over $\mathbb{R}$. A similar description holds when $\kappa=\mathbb{R}$. Here, as before, $\gamma^{\cdot}$ denotes the descending filtration $F^{\cdot} \cap \bar{F}^{\text {. }}$ on $H^{m}\left(X^{\text {an }}, \mathbb{R}\right)$ endowed with its real Hodge structure. The locally-free sheaf $\zeta_{\mathbb{C}}^{\omega}\left(H^{m}(X), \gamma^{\prime}\right)$ has the remarkable description ( $c f$. [13] Thm. 4.4)

$$
\begin{aligned}
\zeta_{\mathbb{C}}^{\omega}\left(H^{m}(X), \gamma^{\prime}\right) & \simeq \operatorname{Ker}\left(\mathbb{R}^{m} \pi_{*}\left(\Omega_{X_{\mathbb{C}}^{\text {an }} \times \mathbb{R} / \mathbb{R}}, s d\right) \rightarrow\left(\mathbb{R}^{m} \pi_{*} \mathcal{D} \mathcal{R}_{X / \mathbb{C}}\right) / \mathcal{T}_{X / \mathbb{C}}\right) \\
H_{\mathrm{ar}}^{m}(X) & =\Gamma\left(\mathbb{R}, \zeta_{\mathbb{C}}^{\omega}\left(H^{m}(X), \gamma^{\prime}\right) \quad \text { if } \quad \kappa=\mathbb{C}\right.
\end{aligned}
$$


where $\pi: X_{\mathbb{C}}^{\text {an }} \times \mathbb{R} \rightarrow \mathbb{R}$ is the projection, $s$ is a standard coordinate on $\mathbb{R}, \mathcal{A}_{\mathbb{R}}$ denotes the sheaf of real-analytic functions on the real analytic manifold $\mathbb{R}, \mathcal{D} \mathcal{R}_{X / \mathbb{C}}$ is the cokernel of the natural inclusion of complexes of $\pi^{-1} \mathcal{A}_{\mathbb{R}}$-modules on $X_{\mathbb{C}}^{\text {an }} \times \mathbb{R}$

$$
\pi^{-1} \mathcal{A}_{\mathbb{R}} \hookrightarrow\left(\Omega_{X_{\mathbb{C}}^{\text {an }} \times \mathbb{R} / \mathbb{R}}, s d\right)
$$

and $\mathcal{T}_{X / \mathbb{C}}$ is the $\mathcal{A}_{\mathbb{R}}$-torsion in $\mathbb{R}^{m} \pi_{*} \mathcal{D} \mathcal{R}_{X / \mathbb{C}} \cdot \Omega_{X_{\mathbb{C}}^{\text {an }} \times \mathbb{R} / \mathbb{R}}$ is the complex of $\mathbb{C}$-valued smooth relative differential forms on $X^{\text {an }} \times \mathbb{R} / \mathbb{R}$ which are holomorphic in the $X^{\text {an }}$-coordinates and real analytic in the $\mathbb{R}$-variable. One considers the scaling flow on $\mathbb{R}$ given by the map $\phi_{\mathbb{C}}^{t}(s)=s e^{-t}$. It induces an action of this group on the relative differential complex by means of: $\psi^{t}(\omega)=e^{t \operatorname{deg} \omega} \cdot\left(\mathrm{id} \times \phi_{\mathbb{C}}^{t}\right)^{*} \omega$. This action defines in turn a $\mathcal{A}_{\mathbb{R}}$-linear action on the complex of higher direct image sheaves on $\mathbb{R}$. A similar

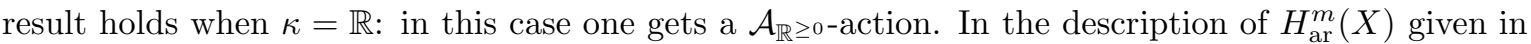
(6.1), the Hodge theoretic notions required in the definition of the archimedean cohomology have been replaced by using suitably deformed complexes of sheaves of modules on $\mathbb{R}$ (on $\mathbb{R}^{\geq 0}$ when $\kappa=\mathbb{R}$ ). The deformed complex of locally free sheaves of relative differentials $\left(\Omega_{X_{\complement} \times \mathbb{A}^{1} / \mathbb{A}^{1}}, z d\right)$, filtered by the Hodge filtration $F$, for $z$ coordinate on $\mathbb{A}^{1}$, was firstly studied by Simpson in [27. He introduced the algebraic version $\zeta_{\mathbb{C}}\left(H^{m}\left(X^{\text {an }}, \mathbb{C}\right), F^{\cdot}\right)$ of the real analytic Rees sheaf and proved that $\zeta_{\mathbb{C}}\left(H^{m}\left(X^{\text {an }}, \mathbb{C}\right), F^{\cdot}\right) \simeq$ $\mathbb{R}^{m} \pi_{*}\left(\Omega_{X_{\mathbb{C} \times \mathbb{A}^{1} / \mathbb{A}^{1}}}, z d\right)$. Following this viewpoint, (6.1) is the analogue of Simpson's formula for the non-algebraic filtration $\gamma^{*}$. A very interesting fact $(c f$. [13, $\S 4)$ is that in the real analytic setting, the higher direct image sheaves fit into short exact sequences of coherent $\mathcal{A}_{\mathbb{R}}$-modules

$$
0 \rightarrow \mathbb{R}^{m} \pi_{*}\left(\pi^{-1} \mathcal{A}_{\mathbb{R}}\right) \rightarrow \mathbb{R}^{m} \pi_{*}\left(\Omega_{X_{\mathbb{C}}^{\text {an }} \times \mathbb{R} / \mathbb{R}}, s d\right) \stackrel{\alpha}{\rightarrow} \mathbb{R}^{m} \pi_{*} \mathcal{D} \mathcal{R}_{X / \mathbb{C}} \rightarrow 0
$$

where $\mathbb{R}^{m} \pi_{*}\left(\pi^{-1} \mathcal{A}_{\mathbb{R}}\right)=H^{m}\left(X^{\text {an }}, \mathbb{R}\right) \otimes \mathcal{A}_{\mathbb{R}}$. We like to think the sheaf $\mathbb{R}^{m} \pi_{*}\left(\Omega_{X_{C}^{\text {an }} \times \mathbb{R} / \mathbb{R}}, s d\right)$ as the archimedean real analytic analog of the relative analytic hypercohomology $\mathbb{R}^{m} f_{*} \Omega_{\mathfrak{X} / \Delta}(\log Y)$ over a small disk $\Delta$ centered at the origin, whose algebraic description has been recalled in $\S 3.3$. It turns out that $\zeta_{\mathbb{C}}^{\omega}\left(H^{m}(X), \gamma^{*}\right)$ is also canonically isomorphic to a twisted dual of $\mathbb{R}^{m} \pi_{*} \mathcal{D} \mathcal{R}_{X / \mathbb{C}}$. More precisely, if $d, m \in \mathbb{Z}_{\geq 0}$ with $m+d=2 n(n=\operatorname{dim} X)$, then there are isomorphisms of $\mathcal{A}_{\mathbb{R}}$-modules $(c f$. 13. Thm. 4.2)

$$
\zeta_{\mathbb{C}}^{\omega}\left(H^{m}(X), \gamma^{\cdot}\right) \simeq(2 \pi \sqrt{-1})^{1-n} \mathcal{H o m}_{\mathcal{A}_{\mathbb{R}}}\left(\mathbb{R}^{d} \pi_{*} \mathcal{D} \mathcal{R}_{X / \mathbb{C}}, \mathcal{A}_{\mathbb{R}}(-n)\right)
$$

The dualizing operation detects the $\gamma^{\prime}$-filtration from the Hodge filtration on $\mathbb{R}^{d} \pi_{*} \mathcal{D} \mathcal{R}_{X / \mathbb{C}}$. From (6.1) and (6.3) one gets isomorphisms respecting the $\mathcal{A}_{\mathbb{R}}$-module structures and the flow

$$
\operatorname{Ker}\left(\mathbb{R}^{m} \pi_{*}\left(\Omega_{X_{\mathbb{C}}^{\text {an }} \times \mathbb{R} / \mathbb{R}}, s d\right) \rightarrow\left(\mathbb{R}^{m} \pi_{*} \mathcal{D} \mathcal{R}_{X / \mathbb{C}}\right) / \mathcal{T}_{X / \mathbb{C}}\right) \simeq(2 \pi \sqrt{-1})^{1-n} \mathcal{H o m}_{\mathcal{A}_{\mathbb{R}}}\left(\mathbb{R}^{d} \pi_{*} \mathcal{D} \mathcal{R}_{X / \mathbb{C}}, \mathcal{A}_{\mathbb{R}}(-n)\right) .
$$

This statement is the dynamical sheaf-theoretic analog of the duality isomorphisms between the hypercohomology of the complexes of vector spaces $\operatorname{Ker}(N)^{\circ}$ and $\operatorname{Coker}(N)^{\circ}$ of 9 (Prop. 4.13) induced by powers of the 'local monodromy at arithmetic infinity', that is, of the duality $S$ of (2.19).

This way, one can reinterpret the archimedean cohomology as the space of global sections on $\mathbb{R}$ (on $\mathbb{R}^{\geq 0}$ when $\left.\kappa=\mathbb{R}\right)$ of the sheaf inverse image in $\mathbb{R}^{m} \pi_{*}\left(\Omega_{X^{\text {an }} \times \mathbb{R} / \mathbb{R}}, s d\right)$ of the maximal $\mathcal{A}_{\mathbb{R}}$-submodule of $\mathbb{R}^{m} \pi_{*} \mathcal{D} \mathcal{R}_{X / \mathbb{C}}$ with support in $0 \in \mathbb{R}$. This statement is in accord with the classical description of the inertia invariants as the kernel of the local monodromy map $N$, viewed as the residue at zero of the Gauss-Manin connection

$$
\operatorname{Ker}\left(\operatorname{Res}_{0} \nabla: \mathbb{R}^{m} f_{*} \Omega_{\mathfrak{X} / \Delta}(\log Y) \otimes_{\mathcal{O}_{\Delta}} k(0) \rightarrow \mathbb{R}^{m} f_{*} \Omega_{\mathfrak{X} / \Delta}(\log Y) \otimes_{\mathcal{O}_{\Delta}} k(0)\right)
$$

cf. $\S 3.3$ and (5.24), (5.25), (5.26) in the archimedean case.

In the archimedean setting $(e . g$. for $\kappa=\mathbb{C})$, the exact sequences

$$
0 \rightarrow \zeta_{\mathbb{C}}^{\omega}\left(H^{m}(X), \gamma^{\cdot}\right) \rightarrow \mathbb{R}^{m} \pi_{*}\left(\Omega_{X_{\mathbb{C}}^{\text {an }} \times \mathbb{R} / \mathbb{R}}, s d\right) \rightarrow\left(\mathbb{R}^{m} \pi_{*} \mathcal{D} \mathcal{R}_{X / \mathbb{C}}\right) / \mathcal{T}_{X / \mathbb{C}} \rightarrow 0
$$

are the sheaf theoretic analogs of the hypercohomology exact sequences associated to the nearby cycles complex as defined by Deligne in [26] ( $c f$. Exp. XIII, § 1.4: (1.4.2.2)). At arithmetic infinity, the hypercohomology of the complex of vanishing cycles is replaced by the hypercohomology sheaf 
$\left(\mathbb{R}^{m} \pi_{*} \mathcal{D} \mathcal{R}_{X / \mathbb{C}}\right) / \mathcal{T}_{X / \mathbb{C}}$, whereas the archimedean analog of the variation map in the formalism of the nearby cycles produces the duality isomorphisms (6.4).

The sequence (6.5) has also interesting analogies with the exact sequence of $C_{k}$-modules $\left(C_{k}=\right.$ idèle class group of a global field $k$ )

$$
0 \rightarrow L_{\delta}^{2}(X)_{0} \stackrel{E}{\rightarrow} L_{\delta}^{2}(X) \rightarrow \mathcal{H} \rightarrow 0
$$

studied by A. Connes in 4, $\S$ III (33). It is tempting to interpret the Pólya-Hilbert space $\mathcal{H}$ as the Hilbert space analogue of the cohomology $\left(\mathbb{R} \cdot \pi_{*} \mathcal{D} \mathcal{R}_{X / \mathbb{C}}\right) / \mathcal{T}_{X / \mathbb{C}}$. This relations suggest a 'singular behavior' of $\overline{\operatorname{Spec}(\mathbb{Z})}$ around infinity.

One of the interesting questions related to the archimedean cohomology and the archimedean factor is that of writing the logarithm $\log L_{\kappa}\left(H^{m}(X), s\right)$ of the regularized determinant (3.15) via a Lefschetz trace formula for the Frobenius operator. To this purpose, a first necessary step seems that of relating the complex $\mathcal{T}$ to a complex of sheaves of (relative) differential forms on $X_{\mathbb{C}}^{\text {an }} \times \mathbb{R}$ and eventually interpreting the Tate twists in the complex $K^{\cdot}$ (the degree in the variable $U$ in $\mathcal{T}^{\cdot}$ ) as "Fourier modes". We hope to develop these topics in a future work.

\section{7. "Arithmetic" SPECTRAL triples}

In this section we present a refined version of the proposed construction of an "arithmetic spectral triple" in [10. This version has the advantage that it holds at the level of differential forms and for $X$ of any dimension. We first introduce natural subcomplexes and quotient complexes of $\mathcal{T}$.

7.1. Inertia invariants and coinvariants. We consider certain complexes of vector spaces related to the action of the endomorphism $N$ on $(\mathcal{T}, \delta)$. We introduce the notation $\mathcal{T}_{\dot{\ell}} \subset \mathcal{T}$ for the $\mathbb{Z}$-graded linear subspace obtained as follows. For fixed $(p, q)$ with $p+q=m$, let $\left(\mathcal{T}_{p, q}^{m, *}\right)_{\ell} \subset \mathcal{T}_{p, q}^{m, *}$ be the $2 \mathbb{Z}$-graded real vector space spanned by elements of the form $\alpha \otimes U^{r} \otimes \hbar^{k}$, with $\alpha \in\left(\Omega_{X}^{p, q} \oplus \Omega_{X}^{q, p}\right)_{\mathbb{R}}$ and $(r, k) \in \Lambda_{p, q}$ lying on the line $k=2 r+m+\ell$ (cf. Figure 3i). We let $\mathcal{T}_{\ell}=\oplus_{p, q}\left(\mathcal{T}_{p, q}^{m, *}\right)_{\ell}$.

Each $\mathcal{T}_{\ell}$ is a subcomplex with respect to the differential $d^{\prime}=\hbar d$, while the second differential satisfies $d^{\prime \prime}: \mathcal{T}_{\ell} \rightarrow \mathcal{T}_{\ell-1}^{+1}$.

Similarly, we denote by $\check{\mathcal{T}}_{\ell}$ the linear subspace of $\mathcal{T}$; which is the direct sum of the subspaces $\left(\check{\mathcal{T}}_{p, q}^{m, *}\right)_{\ell} \subset$ $\mathcal{T}_{p, q}^{m, *}$ spanned by elements $\alpha \otimes U^{r} \otimes \hbar^{k}$, with $\alpha \in\left(\Omega_{X}^{p, q} \oplus \Omega_{X}^{q, p}\right)_{\mathbb{R}}$ and with $(r, k) \in \Lambda_{p, q}$ lying on the horizontal line $k=\ell$ ( $c f$. Figure 3 ). Each $\check{\mathcal{T}}_{\ell}$ is a subcomplex with respect to the differential $d^{\prime \prime}$, while $d^{\prime}: \check{\mathcal{T}}_{\ell} \rightarrow \check{\mathcal{T}}_{\ell+1}^{\cdot+1}$.

In particular, for $\ell=0$ and $k=2 r+m \geq 0$, we obtain the subcomplex of "inertia invariants"

$$
\mathcal{T}_{0}:=\operatorname{Ker}(N)^{\text {. }}
$$

This complex agrees with the complex that computes the $\mathbb{H}^{\cdot}(\mathcal{T} \cdot \delta)^{N=0}$, for all $k=2 r+m>0$, so that the map

is almost always a bijection.

$$
\mathbb{H}^{*}\left(\operatorname{Ker}(N)^{\cdot}, d\right) \rightarrow \mathbb{H}^{*}(\mathcal{T} \cdot d)^{N=0}
$$

Similarly, for $u \in \mathbb{N}$, one can consider subcomplexes $\mathcal{T}_{0, u} \subset \mathcal{T}$.

$$
\mathcal{T}_{0, u}:=\oplus_{\ell=0}^{u} \mathcal{T}_{\ell}=\operatorname{Ker}\left(N^{u+1}\right)^{:}
$$

These satisfy $\mathcal{T} \cdot \lim _{u} \mathcal{T}_{0, u}$. We also consider the quotient complexes

$$
\check{\mathcal{T}}_{0, u}:=\oplus_{\ell=0}^{u} \check{\mathcal{T}}_{\ell}=\operatorname{Coker}\left(N^{u+1}\right),
$$

where we denote by $\check{\delta}$ the induced differential on $\check{\mathcal{T}}_{0, u}$. We obtain this way $\mathcal{T} \cdot \lim _{u} \check{\mathcal{T}}_{0, u}$. We call $\operatorname{Coker}(N)^{\prime}$ the complex of "inertia coinvariant" and we refer to the $\operatorname{Ker}\left(N^{u+1}\right)$ ' and $\operatorname{Coker}\left(N^{u+1}\right)$ ' as higher inertia co/invariants. 


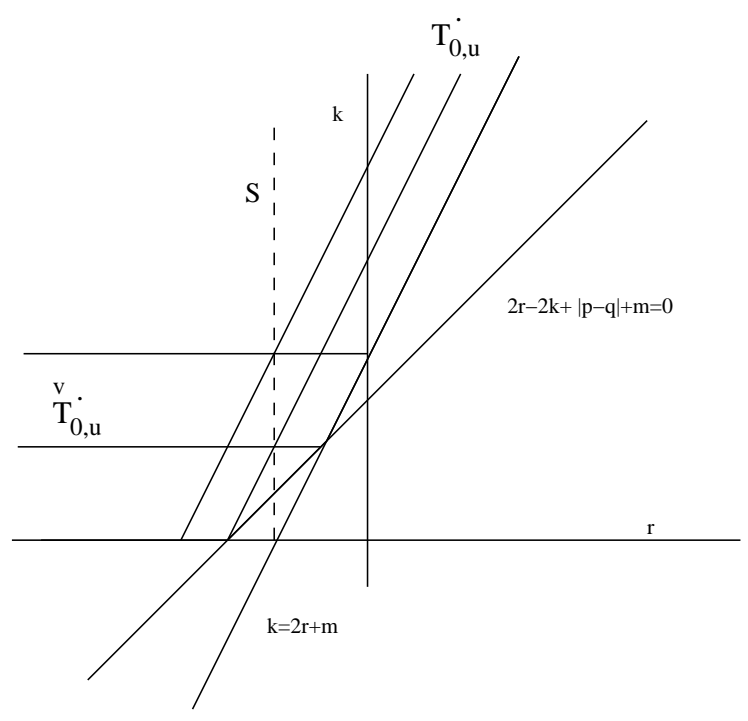

Figure 3. The complexes $\check{\mathcal{T}}_{0, u}$ and $\mathcal{T}_{0, u}$ and the duality $S$

7.2. Spectral triple. We consider the complex

$$
\mathcal{T}_{u}=\mathcal{T}_{0, u} \oplus \check{\mathcal{T}}_{0, u}[+1]
$$

with induced differential $\delta_{u}=\delta \oplus \check{\delta}$, where $\left(\mathcal{T}_{0, u}, \delta\right)$ and $\left(\check{\mathcal{T}}_{0, u}, \check{\delta}\right)$ are the complexes of higher invariants and coinvariants of (7.1) and (7.2).

We still denote by $\Phi$ the linear operator on $\mathcal{T}_{u}$ which agrees with the operator $\Phi=-U \partial_{U}$ on the subspaces $0 \oplus \check{\mathcal{T}}_{0, u}$ and $\mathcal{T}_{0, u} \oplus 0$, identified with the corresponding linear subspaces of $\mathcal{T}$.

On the compact Kähler manifold $X$ consider the operator $d+d^{*}$ on real forms $\Omega_{X, \mathbb{R}}$. For the next result we need to assume the following conditions on the spectrum of $d+d^{*}$ :

$$
\begin{array}{ll}
\#\left\{(r, \lambda) \in \mathbb{Z} \times \operatorname{Spec}\left(d+d^{*}\right): t=r+\lambda\right\}<\infty & \forall t \in \mathbb{R} \\
\left\{r+\lambda: r \in \mathbb{Z}, \lambda \in \operatorname{Spec}\left(d+d^{*}\right)\right\} \subset \mathbb{R} & \text { is discrete. }
\end{array}
$$

Theorem 7.1. Let $U(\mathfrak{g})$ be the universal enveloping algebra of the Lie algebra $\mathfrak{g}=\operatorname{sl}(2, \mathbb{R})$. Let $\mathcal{H}$ ' denote the completion of $\mathcal{T}_{u}$ with respect to the inner product induced by (2.2). Let $\mathcal{D}$ be the linear operator $\mathcal{D}=\Phi+\delta_{u}+\delta_{u}^{*}$. Let $\mathcal{A}=\mathcal{C}^{\infty}(X, \mathbb{R}) \otimes U(\mathfrak{g})$. If $X$ has the property (7.4), then the data $(\mathcal{A}, \mathcal{H}, \mathcal{D})$ satisfy the properties:

- The representation (2.23) determines an action of the algebra $\mathcal{A}$ by bounded operators on the Hilbert space $\mathcal{H}^{\text {. }}$

- The commutators $\left[\mathcal{D}, \sigma^{L}(a)\right]$, for $a \in \mathcal{A}$, are bounded operators on $\mathcal{H}$.

- The operator $\mathcal{D}$ is a densely defined unbounded self-adjoint operator on $\mathcal{H}$, such that $\left(1+\mathcal{D}^{2}\right)^{-1}$ is a compact operator.

Proof. The representation $\sigma^{L}$ of $\operatorname{SL}(2, \mathbb{R})$ on $\mathcal{T}$. defined in 2.23 preserves the subspaces $\mathcal{T}_{0, u}$ and $\check{\mathcal{T}}_{0, u}$. In fact, the operator $\mathbb{L}$ changes $m \mapsto m+2, r \mapsto r-1$ and $k \mapsto k$, so that the constraint $k \leq u$ defining $\check{\mathcal{T}}_{0, u}$ and the constraint $k=2 r+m+\ell$ with $0 \leq \ell \leq u$ defining $\mathcal{T}_{0, u}$ are preserved by the action of $\mathbb{L}$. Similarly, the involution $\tilde{S}$ changes $m \mapsto 2 n-m, r \mapsto r-(n-m)$ and $k \mapsto k$, so that again both constraints $k \leq u$ and $k=2 r+m+\ell$, for $0 \leq \ell \leq u$, are preserved. Thus, we can consider on $\mathcal{T}_{u}$ the corresponding derived representation $d \sigma^{L}$ of the Lie algebra $\mathfrak{g}=\operatorname{sl}(2, \mathbb{R})$,

$$
d \sigma^{L}(v)=\left.\frac{d}{d s} \sigma^{L}(\exp (s v))\right|_{s=0}
$$


Let $\left\{v_{ \pm}, v_{0}\right\}$ be the basis of $\mathfrak{g}$ with $\left[v_{0}, v_{+}\right]=2 v_{+},\left[v_{0}, v_{-}\right]=-2 v_{-}$, and $\left[v_{+}, v_{-}\right]=v_{0}$. We have $d \sigma^{L}\left(v_{+}\right)=\mathbb{L}$, while $d \sigma^{L}\left(v_{0}\right)$ is the linear operator that multiplies elements $\alpha \otimes U^{r} \otimes \hbar^{k}$ with $\alpha \in \Omega_{X, \mathbb{R}}^{m}$ by $-n+m$. Thus, we obtain an action of the algebra $U(\mathfrak{g})$ on $\mathcal{H}$ by bounded linear operators.

We have $\left[\sigma^{L}(\gamma), \delta_{u}\right]=\left[\sigma^{L}(\gamma), \delta_{u}^{*}\right]=0$ hence $\left[\mathcal{D}, d \sigma^{L}(v)\right]=\left[\Phi, d \sigma^{L}(v)\right]$. Using $[\Phi, \mathbb{L}]=\mathbb{L}$ and $[\Phi, \tilde{S}]=$ $(-n+m) \tilde{S}$, we obtain that $\left[\mathcal{D}, d \sigma^{L}(v)\right]$ is a bounded operator for all $v \in U(\mathfrak{g})$. The algebra of real valued smooth functions $\mathcal{C}^{\infty}(X, \mathbb{R})$ acts on $\mathcal{T}$ and on $\mathcal{T}_{u}$ by the usual action on real forms $\Omega_{X, \mathbb{R}}$. This action commutes with $\Phi$ so that $[\mathcal{D}, f]=\left[\delta_{u}+\delta_{u}^{*}, f\right]$, for all $f \in \mathcal{C}^{\infty}(X, \mathbb{R})$, and we can estimate $\|[\mathcal{D}, f]\| \leq C \sup |d f|$ for some $C>0$.

For $\mathcal{D}_{u}=\delta_{u}+\delta_{u}^{*}$, we have $\mathcal{D}=\Phi+\mathcal{D}_{u}$, with $\left[\Phi, \mathcal{D}_{u}\right]=0$. The operator $\Phi$ on $\mathcal{T}_{u}$ has spectrum $\mathbb{Z}$. The eigenspace $E_{r}$ with eigenvalue $r \in \mathbb{Z}$ is the span of the elements

$$
\left(\alpha_{1} \otimes U^{r} \otimes \hbar^{k_{1}}, \alpha_{2} \otimes U^{r} \otimes \hbar^{k_{2}}\right) \in \mathcal{T}_{0, u} \oplus \check{\mathcal{T}}_{0, u},
$$

namely, elements with $\alpha_{i} \in \Omega_{X, \mathbb{R}}^{m_{i}}$ and $k_{i}$ satisfying $0 \leq k_{1}-2 r-m_{1} \leq u$ and $0 \leq k_{2} \leq u$. The operator $\mathcal{D}_{u}$ restricted to the eigenspace $E_{r}$ has discrete spectrum. The multiplicity $m_{\lambda}$ of an eigenvalue $\lambda$ of $\left.\mathcal{D}_{u}\right|_{E_{r}}$ is bounded by $2 u n_{\lambda}$, where $n_{\lambda}$ is the multiplicity of $\lambda$ as an eigenvalue of the operator $d+d^{*}$ on real differential forms $\Omega_{X, \mathbb{R}}$. Condition (7.4) then implies that $\mathcal{D}$ has discrete spectrum with finite multiplicities and that $\left(1+\mathcal{D}^{2}\right)^{-1}$ is compact.

When passing to cohomology, we obtain an induced structure of the following form.

Corollary 7.2. Let now $\mathcal{H}$ denote the Hilbert completion of the cohomology $\mathbb{H}^{\cdot}\left(\mathcal{T}_{u}^{\cdot}, \delta_{u}\right)$ and let $\mathcal{D}=\Phi$, the operator induced in cohomology. For $\mathcal{A}=U(\mathfrak{g})$, the triple $(\mathcal{A}, \mathcal{H}, \mathcal{D})$ satisfies:

- The representation (2.23) induces an action of algebra $\mathcal{A}$ by bounded operators on $\mathcal{H}$.

- The commutators $\left[\mathcal{D}, \sigma^{L}(a)\right]$, for $a \in \mathcal{A}$, are bounded operators on $\mathcal{H}$.

- The operator $\mathcal{D}$ is a densely defined unbounded self-adjoint operator on $\mathcal{H}$, such that $\left(1+\mathcal{D}^{2}\right)^{-1}$ is a compact operator.

Proof. The statement follows as in the case of Theorem 7.1 Notice that now we have $\operatorname{Spec}(\Phi)=\mathbb{Z}$ with multiplicites

$$
m_{r}=\operatorname{dim} \frac{\operatorname{Ker}\left(d^{\prime}: \mathcal{T}_{0, u}^{\cdot, 2 r} \rightarrow \mathcal{T}_{0, u}^{\cdot+1,2 r}\right)}{\operatorname{Im}\left(d^{\prime}: \mathcal{T}_{0, u}^{--1,2 r} \rightarrow \mathcal{T}_{0, u}^{\cdot, 2 r}\right)}+\operatorname{dim} \frac{\operatorname{Ker}\left(d^{\prime \prime}: \check{\mathcal{T}}_{0, u}^{+1,2 r} \rightarrow \check{\mathcal{T}}_{0, u}^{+2,2 r}\right)}{\operatorname{Im}\left(d^{\prime \prime}: \check{\mathcal{T}}_{0, u}^{\cdot, 2 r} \rightarrow \check{\mathcal{T}}_{0, u}^{++1,2 r}\right)}
$$

This cohomological result no longer depends on the property (7.4).

Recall that $(\mathcal{A}, \mathcal{H}, \mathcal{D})$ is a spectral triple in the sense of Connes (cf. [6]) if the three properties listed in the statement of Theorem [7.1 and Corollary 7.2 hold and the algebra $\mathcal{A}$ is a dense involutive subalgebra of a $C^{*}$-algebra.

In our case, the adjoints of elements in $\mathcal{A}$ with respect to the inner product on $\mathcal{H}$ ' are again contained in $\mathcal{A}$. In fact, one can see that the adjoint of the Lefschetz $\mathbb{L}$ is given by

$$
\mathbb{L}^{*}=(\cdot \mid \omega) U,
$$

where $\rfloor$ is the interior product and $\omega$ is the Kähler form, and we obtain (7.5) from

$$
\sigma^{L}(w)^{-1} \mathbb{L} \sigma^{L}(w)=*((* \cdot) \wedge \omega) U .
$$

Moreover, a choice of the Kähler form (of the Kähler class in the cohomological case) determines a corresponding representation of the involutive algebra $\mathcal{A}$ on the Hilbert space $\mathcal{H}$. The choice of the Kähler class ranges over the Kähler cone

$$
\mathcal{K}=\left\{c \in H^{1,1}(X): \int_{M} c^{k}>0\right\}
$$


for all $M \subset X$ complex submanifolds of dimension $1 \leq k \leq X$. Thus, in the case of Corollary [7.2 for instance we can consider a norm

$$
\|a\|:=\sup _{c \in \overline{\mathcal{K}}:\|c\|=1}\left\|\sigma_{c}^{L}(a)\right\|
$$

where $\overline{\mathcal{K}}$ is the nef cone and $\sigma_{c}^{L}$ is the representation of $\mathcal{A}$ determined by the choice of the class $c$. Thus, the data $(\mathcal{A}, \mathcal{H}, \mathcal{D})$ of Theorem 7.1 and Corollary 7.2 determine a spectral triple.

An interesting arithmetic aspect of spectral triples is that they have an associated family of zeta functions, the basic one being the zeta function of the Dirac operator,

$$
\zeta_{\mathcal{D}}(z)=\operatorname{Tr}\left(|\mathcal{D}|^{-z}\right)=\sum_{\lambda} \operatorname{Tr}(\Pi(\lambda,|\mathcal{D}|)) \lambda^{-z}
$$

where $\Pi(\lambda,|\mathcal{D}|)$ denotes the orthogonal projection onto the eigenspace $E(\lambda,|\mathcal{D}|)$. More generally, one considers for $a \in \mathcal{A}$ the corresponding zeta function

$$
\zeta_{a, \mathcal{D}}(z)=\operatorname{Tr}\left(a|\mathcal{D}|^{-z}\right)=\sum_{\lambda} \operatorname{Tr}(a \Pi(\lambda,|\mathcal{D}|)) \lambda^{-z} .
$$

These provide a refined notion of dimension for noncommutative spaces, the dimension spectrum, which is the complement in $\mathbb{C}$ of the set where all the $\zeta_{a, \mathcal{D}}$ extend holomorphically.

One can also consider the associated two-variable zeta functions,

$$
\zeta_{a, \mathcal{D}}(s, z):=\sum_{\lambda} \operatorname{Tr}(a \Pi(\lambda,|\mathcal{D}|))(s-\lambda)^{-z}
$$

and the corresponding regularized determinants,

$$
\operatorname{det}_{\infty a, \mathcal{D}}(s):=\exp \left(-\left.\frac{d}{d z} \zeta_{a, \mathcal{D}}(s, z)\right|_{z=0}\right) .
$$

Proposition 3.3 then shows that the archimedean factor of the Hasse-Weil L-function is given by the regularized determinant of a zeta function $\zeta_{a, \mathcal{D}}$ of the spectral triple of Corollary 7.2 namely the one for $a=\sigma^{L}(w)^{2}$. One advantage of this point of view is that one can now see the archimedean factor of the Hasse-Weil L-function as an element in the family $\operatorname{det}_{\infty a, \mathcal{D}}$ associated to the noncommutative geometry $(\mathcal{A}, \mathcal{H}, \mathcal{D})$.

Different representations of the Lie algebra $\mathfrak{g}=\operatorname{sl}(2)$, for different choice of the Kähler class and the corresponding Lefschetz operators, were considered also in [20. It would be interesting to see if one can use this formalism of spectral triples in that context to further investigate the structure of the resulting Kähler Lie algebra (or of the Neron-Severi Lie algebra of projective varieties considered in [20]).

In the special case of arithmetic surfaces considered in [10, where $X$ is a compact Riemann surface, the result of Corollary 7.2 can be related to the "arithmetic spectral triple" of [10].

Consider the complex $\operatorname{Cone}(N)^{\cdot}=\mathcal{T} \cdot \oplus \mathcal{T} \cdot[+1]$ with differential

$$
d_{\text {Cone }}=\left(\begin{array}{cc}
\delta & N \\
0 & -\delta
\end{array}\right)
$$

Proposition 2.23 of [10] and $\S 4$ of [9] show that, for $X$ a compact Riemann surface, we have

$$
\mathbb{H}^{\cdot}\left(\operatorname{Cone}(N)^{\prime}, d_{\text {Cone }}\right) \simeq \mathbb{H}^{\cdot}\left(\operatorname{Ker}(N)^{\cdot}, d^{\prime}\right) \oplus \mathbb{H}^{\cdot+1}\left(\operatorname{Coker}(N)^{\prime}, d^{\prime \prime}\right) .
$$

This is isomorphic to $\left.\mathbb{H}^{\cdot}\left(\mathcal{T}_{u}, \delta_{u}\right)\right|_{u=0}$. Moreover, under this identification, the operator $\Phi$ on the cohomology $\mathbb{H}^{\cdot}\left(\right.$ Cone $\left.(N)^{\prime}, d_{\text {Cone }}\right)$ considered in [10] agrees with the operator $\Phi$ on $\left.\mathbb{H}^{\cdot}\left(\mathcal{T}_{u}, \delta_{u}\right)\right|_{u=0}$. 


\section{Analogies With LOOP SPACE GeOMetry}

Besides the original motivating analogy with the case of a geometric degeneration and the resolution (4.6) of the complex of nearby cycles, the cohomology theory at arithmetic infinity defined by the complex $(\mathcal{T}, \delta)$ also bears some interesting formal analogies with Givental's homological geometry on the loop space of a Kähler manifold ( $c f$. [15]).

Let $X$ be a compact Kähler manifold, with the symplectic form $\omega$ representing an integral class in cohomology, such that the morphism $\omega: \pi_{2}(X) \rightarrow \mathbb{Z}$ is onto. Let $L X$ denote the space of contractible loops on $X$, and $\widetilde{L X}$ the cyclic cover with group of deck transformations

$$
\pi_{2}(X) / \operatorname{Ker}\left\{\omega: \pi_{2}(X) \rightarrow \mathbb{Z}\right\} \cong \mathbb{Z}
$$

and with the $S^{1}$-action that rotates loops. This covering makes the action functional

$$
\mathcal{A}(\phi)=\int_{\Delta} \phi^{*} \omega, \quad \forall \phi \in \widetilde{L X}
$$

single valued, for $\partial \Delta=S^{1}$. In fact, if $\gamma$ denotes the generator of (18.1), we have $\gamma^{*} \mathcal{A}=\mathcal{A}+1$. The critical manifold $\operatorname{Crit}(\mathcal{A})=F i x\left(S^{1}\right)$ consists of a trivial cyclic cover of the submanifold of constant loops $X$. Formally, one can consider an equivariant Floer complex for the functional $\mathcal{A}$, which is the complex (2.1) with a differential $d_{S^{1}} \pm \pi_{*} \pi^{*}$, which combines the equivariant differential on each component of $\operatorname{Crit}(\mathcal{A})$ with a pullback-pushforward along gradient flow lines of $\mathcal{A}$ between different components of the critical manifold.

More precisely, a formal setting for the construction of equivariant Floer cohomologies can be described as follows. On a configuration space $\mathcal{C}$, which is an infinite dimensional manifold with an $S^{1}$ action, consider an $S^{1}$-invariant functional $\mathcal{A}: \mathcal{C} \rightarrow \mathbb{R}$, satisfying the following assumptions: $(i)$ The critical point equation $\nabla \mathcal{A}=0$ cuts out a finite dimensional smooth compact $S^{1}$-manifold $\operatorname{Crit}(\mathcal{A}) \subset \mathcal{C}$. (ii) The Hessian $H(\mathcal{A})$ on $\operatorname{Crit}(\mathcal{A})$ is non-degenerate in the normal directions. (iii) For any $x, y \in \operatorname{Crit}(\mathcal{A})$, there is a well defined locally constant relative index $\operatorname{ind}(x)-\operatorname{ind}(y) \in \mathbb{Z}$. (iv) For any two $S^{1}$-orbits $\mathcal{O}^{ \pm}$in $\operatorname{Crit}(\mathcal{A})$, the set $\mathcal{M}\left(\mathcal{O}^{+}, \mathcal{O}^{-}\right)$of solutions to the flow equation

$$
\frac{d}{d t} u(t)+\nabla \mathcal{A}(u(t))=0, \quad \lim _{t \rightarrow \pm \infty} u(t) \in \mathcal{O}^{ \pm},
$$

modulo reparameterizations by translations, is either empty or a smooth manifold of dimension $\operatorname{ind}\left(\mathcal{O}^{+}\right)-\operatorname{ind}\left(\mathcal{O}^{-}\right)+\operatorname{dim} \mathcal{O}^{+}-1$. (v) The manifolds $\mathcal{M}\left(\mathcal{O}^{+}, \mathcal{O}^{-}\right)$admit a compactification to smooth manifolds with corners, with codimension one boundary strata $\cup_{\operatorname{ind}\left(\mathcal{O}^{+}\right) \geq \operatorname{ind}(\mathcal{O}) \geq \operatorname{ind}\left(\mathcal{O}^{-}\right)} \mathcal{M}\left(\mathcal{O}^{+}, \mathcal{O}\right) \times \mathcal{O}$ $\mathcal{M}\left(\mathcal{O}, \mathcal{O}^{-}\right)$, and with compatible endpoint fibrations $\pi_{ \pm}: \mathcal{M}\left(\mathcal{O}^{+}, \mathcal{O}^{-}\right) \rightarrow \mathcal{O}^{ \pm}$.

For each component $\mathcal{O} \subset \operatorname{Crit}(\mathcal{A})$ one can then consider the $S^{1}$-equivariant de Rham complex

$$
\Omega_{S^{1}}^{\cdot,}(\mathcal{O}):=\Omega_{i n v}(\mathcal{O}) \otimes \mathbb{C}\left[U, U^{-1}\right]
$$

where $U$ is of degree two, so that the total degree of $\alpha \otimes U^{r}$, with $\alpha \in \Omega_{\mathcal{O}}^{m}$ is $i=m+2 r$. The differential is of the form

$$
d_{S^{1}}\left(\alpha \otimes U^{r}\right)=d \alpha \otimes U^{r}+\iota_{V}(\alpha) \otimes U^{r+1}, \quad d_{S^{1}} U=0
$$

where $\iota_{V}$ denotes contraction with the vector field generated to the $S^{1}$-action on $\mathcal{O}$. The complex (8.4) computes the periodic $S^{1}$-equivariant cohomology of $\mathcal{O}$,

$$
H^{\cdot}\left(\Omega_{S^{1}}^{\cdot, \infty}(\mathcal{O}), d_{S^{1}}\right)=H_{S^{1}, p e r}^{\cdot}(\mathcal{O} ; \mathbb{C}),
$$

which is the localization of $H_{S^{1}}(\mathcal{O} ; \mathbb{C})$ obtained by inverting $U$. The Floer complex is then defined as

$$
C F_{S^{1}}^{\ell, \infty}=\oplus_{\ell=\operatorname{ind}(\mathcal{O})+m+2 r} \Omega_{S^{1}}^{m+2 r, \infty}(\mathcal{O}),
$$


with the relative index ind $(\mathcal{O})$ computed with respect to a fixed base point in $\operatorname{Crit}(\mathcal{A})$, and with the Floer differential given by

$$
D_{\mathcal{O}^{+}, \mathcal{O}^{-}}\left(\alpha \otimes U^{r}\right)= \begin{cases}d_{S^{1}}\left(\alpha \otimes U^{r}\right) & \mathcal{O}^{+}=\mathcal{O}^{-} \\ (-1)^{m}\left(\pi_{+*} \pi_{-}^{*} \alpha\right) \otimes U^{r} & \operatorname{ind}\left(\mathcal{O}^{+}\right) \geq \operatorname{ind}\left(\mathcal{O}^{-}\right) \\ 0 & \text { otherwise },\end{cases}
$$

where $\pi_{ \pm}: \mathcal{M}\left(\mathcal{O}^{+}, \mathcal{O}^{-}\right) \rightarrow \mathcal{O}^{ \pm}$are the endpoint projections. The (periodic) equivariant Floer cohomology is the $\mathbb{C}\left[U, U^{-1}\right]$ module

$$
H F_{S^{1}}^{\cdot,}(\mathcal{C} ; \mathcal{A}):=H^{\cdot}\left(C F_{S^{1}}^{\infty}, D\right)
$$

The property $D^{2}=0$ for the Floer differential holds because of the structure of the compactification of the spaces $\mathcal{M}\left(\mathrm{O}^{+}, \mathcal{O}^{-}\right)$and its compatibility with the endpoint fibrations (cf. [2]). The periodic equivariant Floer cohomology is related to equivariant Floer cohomology and homology via a natural exact sequence of complexes ( $c f .[22)$, of the form

$$
0 \rightarrow C F_{S^{1}}^{*,+} \rightarrow C F_{S^{1}}^{*, \infty} \rightarrow C F_{S^{1}}^{*,-} \rightarrow 0,
$$

where $C F_{S^{1}}^{*++}$ is defined as in (8.6), but with $\mathbb{C}[U]$ instead of $\mathbb{C}\left[U, U^{-1}\right]$ in (8.4). The equivariant Floer cohomology is defined as

$$
H F_{S^{1}}^{*}(\mathcal{C} ; \mathcal{A})=H F_{S^{1}}^{*,+}(\mathcal{C} ; \mathcal{A})=H^{*}\left(C F_{S^{1}}^{*,+}, D\right),
$$

while the quotient complex $C F_{S^{1}}^{*,-}$, with the induced Floer differential $D^{-}$, computes the equivariant Floer homology

$$
H F_{*, S^{1}}(\mathcal{C} ;-\mathcal{A})=H F_{S^{1}}^{*,-}(\mathcal{C} ; \mathcal{A}) .
$$

There is also, in Floer theory, an analog of the weight filtration $W$. given by the increasing filtration of the complex (8.6) by index of critical orbits, $\operatorname{ind}(\mathcal{O}) \geq k$,

$$
W_{k} C F^{\ell, \infty}(\mathcal{C}, \mathcal{A})=\oplus_{\operatorname{ind}(\mathcal{O}) \geq k, i+\operatorname{ind}(\mathcal{O})=\ell} \Omega_{S^{1}}^{i}(\mathcal{O}) .
$$

In the cases where the boundary components corresponding to flow lines in $\mathcal{M}\left(\mathcal{O}^{+}, \mathcal{O}^{-}\right)$vanish, the exact sequence collapses and the Floer cohomology is the equivariant cohomology of the critical set, cf. [15] and [16. We have then an analog, in this context, of the Picard-Lefschetz filtration in the form (4.12), by setting

$$
A F^{s, k}:=C F_{S^{1}}^{s+k+1, \infty} / W_{k} C F_{S^{1}}^{s+k+1, \infty},
$$

as the analog of (4.6), and

$$
L_{\ell} A F^{s, k}=W_{2 k+\ell+1} C F_{S^{1}}^{s+k+1, \infty} / W_{k} C F_{S^{1}}^{s+k+1, \infty},
$$

with

$$
g r_{\ell}^{L} A F^{s, k}= \begin{cases}g r_{2 k+\ell+1}^{W} C F_{S^{1}}^{s+k+1, \infty} & \ell+k \geq 0 \\ 0 & \ell+k<0\end{cases}
$$

where

$$
g r_{2 k+\ell+1}^{W} C F_{S^{1}}^{s+k+1, \infty}=\oplus_{\operatorname{ind}(\mathcal{O})=2 k+\ell+1, s-i=\ell+k} \Omega_{S^{1}}^{i}(\mathcal{O}) .
$$

In the case of the loop space of a smooth compact symplectic manifold $X$, the action functional (8.2) is degenerate, the transversality conditions fail and the setup of Floer theory becomes more delicate $c f$. 23]. The argument of [16] shows that, in the case of the loop space of a Kähler manifold, the components $\mathcal{M}\left(\mathcal{O}^{+}, \mathcal{O}^{-}\right)$contribute trivially to the Floer differential, hence the equivariant Floer cohomology is computed by the $E_{1}$ term of the spectral sequence associated to the filtration $W$., namely by the infinite dimensional vector space $H^{\cdot}(X ; \mathbb{C}) \otimes \mathbb{C}\left[U, U^{-1}\right] \otimes \mathbb{C}\left[\hbar, \hbar^{-1}\right]$, where $\hbar$ implements the action of $\mathbb{Z}$ in 8.1 .

Thus, one can see a formal analogy between the complex $\mathfrak{C}$ of (2.9) and an equivariant Floer complex on the loop space. The cutoff $k \geq 0$ is then interpreted as a filtration by sublevel sets, corresponding to considering $H F_{S^{1}}^{\cdot}$ of $\mathcal{C}_{0}=\mathcal{A}^{-1}\left(\mathbb{R}_{\geq 0}\right)$. The cutoff $2 r+m \geq 0$ instead corresponds in this analogy to 
a cutoff on the total degree of the equivariant differential forms, $H F_{S^{1}}^{\cdot \geq 0}$. Finally, the cutoff $k \geq r+q$ in (2.4) can be compared to a splitting of the form (8.9), adapted to the Hodge filtration. As in the case of the analogy with the resolution of the complex of nearby cycles, also in this analogy with Floer theory on loop spaces there is however a fundamental difference in the differentials. In fact, the term $d_{S^{1}}$ of the Floer differential is replaced in the theory at arithmetic infinity by $\hbar d+d^{\prime \prime}$, which would not give a degree one differential on the Floer complex (except in special cases, like hyperkähler manifolds, where all components of $\operatorname{Crit}(\mathcal{A})$ have relative index zero).

By the results of Givental, the equivariant Floer cohomology of the loop space also has an action of the ring $\mathcal{D}$ of differential operators on $\mathbb{C}^{*}$, where $Q$ acts as $\hbar=\gamma^{*}$ and $P$ as a combination of the symplectic form and action functional on the loop space, 15. This structure on the Floer cohomology plays an important role in the phenomenon of mirror symmetry. It is interesting to remark that there is a conjectural mirror relation between the monodromy and the Lefschetz operators ( $c f$. [17] [21]). Thus, the question of developing a more precise relation between the complex $(\mathcal{T} \cdot \delta)$ and Floer theory, with the actions (2.27) of the ring of differential operators on $\mathbb{C}^{*}$, may be interesting in this respect, in view of the possibility of addressing such mirror symmetry questions in the context of arithmetic geometry, by adapting to arithmetic cohomological constructions the setting of homological geometry on loop spaces.

\section{REFERENCES}

[1] D.V. Anosov, A.A. Bolibruch, The Riemann-Hilbert problem, Aspects of Mathematics Vol.22, Vieweg, 1994.

[2] D.M. Austin, P.J. Braam, Morse-Bott theory and equivariant cohomology, Floer Memorial Volume, Birkhäuser 1995.

[3] J. Burgos, Arithmetic Chow rings and Deligne-Beilinson cohomology, J.Alg.Geom. 6 (1997) N.2 335-377.

[4] A. Connes, Trace formula in noncommutative geometry and zeros of the Riemann zeta function, Selecta Math. (N.S.) 5 (1999), no. 5, 29-106.

[5] A. Connes, Symetries Galoisiennes et Renormalisation, preprint math.QA/0211199

[6] A. Connes, Geometry from the spectral point of view. Lett. Math. Phys. 34 (1995), no. 3, 203-238.

[7] A. Connes, D. Kreimer, Renormalization in quantum field theory and the Riemann-Hilbert problem. II. The $\beta$ function, diffeomorphisms and the renormalization group. Comm. Math. Phys. 216 (2001), no. 1, 215-241.

[8] A. Connes, M. Marcolli, From physics to number theory via noncommutative geometry, in preparation.

[9] C. Consani, Double complexes and Euler L-factors, Compositio Math. 111 (1998), 323-358.

[10] C. Consani, M. Marcolli, Noncommutative geometry, dynamics, and $\infty$-adic Arakelov geometry, to appear in Selecta Mathematica.

[11] C. Deninger On the $\Gamma$-factors attached to motives, Invent. Math. 104 (1991) 245-261.

[12] C. Deninger Local L-factors of motives and regularized determinants, Invent. Math. 107 (1992) 135-150.

[13] C. Deninger On the $\Gamma$-factors of motives II, Doc. Math. 6 (2001), 69-97.

[14] S.I. Gelfand, Yu.I. Manin, Homological algebra, EMS Vol.38, Springer Verlag, 1999.

[15] A.B. Givental, Homological geometry. I. Projective hypersurfaces. Selecta Math. (N.S.) 1 (1995), no. 2, 325-345.

[16] A.B. Givental, B. Kim, Quantum cohomology of flag manifolds and Toda lattices. Comm. Math. Phys. 168 (1995), no. $3,609-641$

[17] V. Golyshev, V. Lunts, D. Orlov, Mirror symmetry for abelian varieties, J. Algebraic Geom. 10 (2001), no. 3, 433-496.

[18] F. Guillén, V. Navarro Aznar, Sur le théorème local des cycles invariants. Duke Math. J. 61 (1990), no. 1, $133-155$.

[19] S. Lang, $\mathrm{SL}_{2}(\mathbb{R})$, Addison-Wesley, 1975.

[20] E. Looijenga, V.A. Lunts, A Lie algebra attached to a projective variety, Invent. Math. 129 (1997) 361-412.

[21] Yu.I. Manin, Moduli, motives, mirrors, Progress in Matematics Vol. 201, Birkhäuser 2001, pp. 53-73.

[22] M. Marcolli, B.L. Wang, Variants of equivariant Seiberg-Witten Floer homology, preprint math.GT/0211238

[23] S.Piunikhin, D.Salamon, M.Schwarz, Symplectic Floer-Donaldson theory and quantum cohomology. Contact and symplectic geometry (Cambridge, 1994), 171-200, Publ. Newton Inst., 8, Cambridge Univ. Press, Cambridge, 1996.

[24] M. Saito, Modules de Hodge Polarisable. Publ. Res. Inst. Math. Sci. 24 (1988) 849-995.

[25] J. P. Serre, Facteurs locaux des fonctions zêta des variétés algébriques (définitions et conjectures). Sém. DelangePisot-Poitou, exp. 19, 1969/70.

[26] P. Deligne, Groupes de Monodromie en Géométrie Algébrique. Lecture Notes in Mathematics 340, Springer-Verlag, New York 1973

[27] C. Simpson, The Hodge filtration on nonabelian cohomology, Proc. Symp. Pure Math. 62 (2) (1997) $217-281$.

[28] J. Steenbrink, Limits of Hodge structures. Invent. Math. 31 (1976), 229-257.

[29] R.O. Wells, Differential analysis on complex manifolds, Springer Verlag, 1980. 
C. Consani: University of Toronto Canada

E-mail address: kc@math.toronto.edu

M. Marcolli: MaX-Planck Institut für Mathematik Bonn Germany

E-mail address: marcolli@mpim-bonn.mpg.de 